RAPHAEL LUIZ HAIKEL JÚNIOR

\title{
Avaliação Inicial de um Programa de Detecção \\ Precoce do Câncer de Mama, por meio de mamografia, na Região de Barretos
}

Tese apresentada à Faculdade de Medicina da Universidade de São Paulo para obtenção do título de Doutor em Ciências

Área de concentração: Oncologia

Orientador: Prof. Dr. Nestor de Barros

São Paulo

2010 
Dados Internacionais de Catalogação na Publicação (CIP)

Preparada pela Biblioteca da

Faculdade de Medicina da Universidade de São Paulo

(reprodução autorizada pelo autor

Haikel Júnior, Raphael Luiz

Avaliação inicial de um Programa de Deteç̧ão Precoce do Câncer de Mama, por meio de mamografia na região de Barretos / Raphael Luiz Haikel Júnior. -- São Paulo, 2010.

Tese(doutorado)--Faculdade de Medicina da Universidade de São Paulo. Departamento de Radiologia.

Área de concentração: Oncologia.

Orientador: Nestor de Barros.

Descritores: 1.Neoplasias da mama 2.Programas de rastreamento 3.Prevenção de câncer de mama 4.Detecção precoce de câncer 5.Estádio clínico

USP/FM/SBD-116/10 


\section{DEDICATÓRIA}

Neste momento tão especial da vida gostaria de dedicar esta obra, e as incontáveis horas que ela consumiu, à minha família, minha mãe Janete meus irmãos Kiko e Fernanda, e claro sem esquecer a grande guerreira, minha sempre presente minha esposa Luciana que junto com a outra estrela da minha vida, a minha filha Helena, será sempre a força para acordar a cada novo dia. Gostaria de dedicar ao meu pai, Dr. Raphael Luiz Haikel Jr. que sempre serviu e servirá o Hospital, ele soube esperar, aprender e ensinar tudo no seu devido tempo, e neste mesmo tempo também soube abrir mão da minha companhia, no dia a dia, ombro a ombro no atendimento às pacientes para que eu pudesse me lançar em outros caminhos. E como o ditado fala: "quando o aluno está pronto, o mestre aparece" e, portanto gostaria de dedicar este trabalho ao Dr Edmundo Carvalho Mauad, que sempre deu seu apoio, atenção conhecimento e estava sempre pronto a ouvir as agruras do ansioso pupilo. 


\section{AGRADECIMENTOS}

Ao Dr. Paulo Prata e à Drª. Scylla Duarte Prata, idealizadores do Hospital São Judas Tadeu da Fundação PIO XII de Barretos, eles foram e ainda são nossos alicerces.

Ao Sr. Henrique Duarte Prata, pela busca incessante do conhecimento e avanços em todos os setores da Instituição.

Ao Prof. Dr. Nestor de Barros, pela oportunidade de desenvolver este trabalho e poder participar deste universo acadêmico que fascina a todos.

Ao Prof. Dr. Ricardo Renzo Brentani, pelo apoio ao programa de pós-graduação onde podemos canalizar todo o nosso potencial assistencial num grande desenvolvimento cientifico.

À Fundação PIO XII de Barretos, pela possibilidade de realização deste trabalho.

À Universidade de São Paulo - Instituto de Radiologia programa de Pos Graduação em Oncologia, pela oportunidade de realização deste estudo. E aos seus funcionários, sempre prontos a elucidar as nossas dúvidas.

Ao Biólogo MSc Thiago Buosi Silva pela colaboração incondicional na realização do trabalho

Ao Prof. Dr. Adhemar Longatto Filho, pelo apoio na redação do manuscrito e do artigo.

Aos colegas do Departamento de Prevenção, René Aloísio da Costa Vieira, Jacó Saraiva de Castro Mattos, Carlos Eduardo Goulart Silveira, André Luiz Silveira, Julio César Possati Resende pelo incentivo à realização do trabalho.

Aos funcionários do Departamento de Prevenção pela ajuda e auxilio em todas as horas. 


\section{SUMÁRIO}

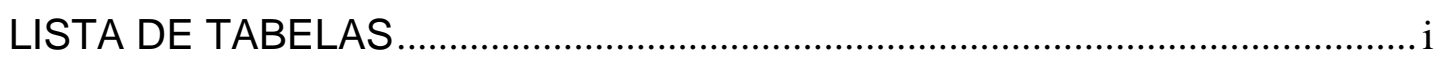

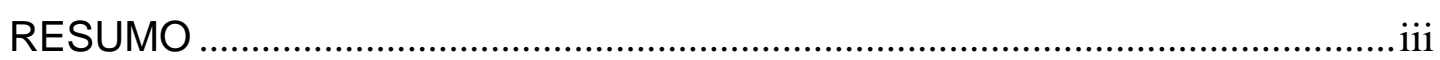

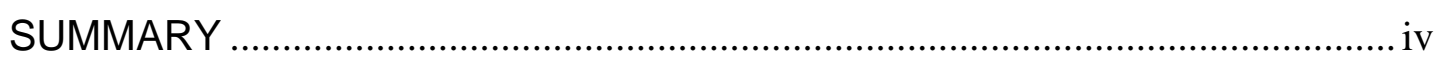

1. INTRODUÇÃO

2. REVISÃO BIBLIOGRÁFICA …………………………………………….... 6

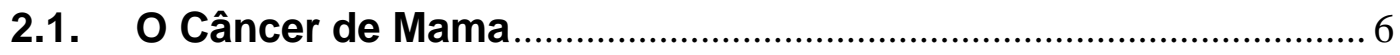

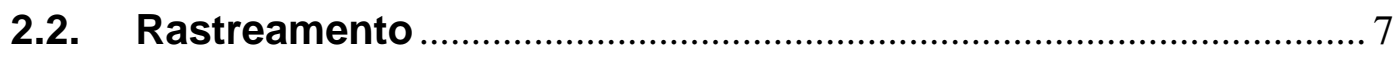

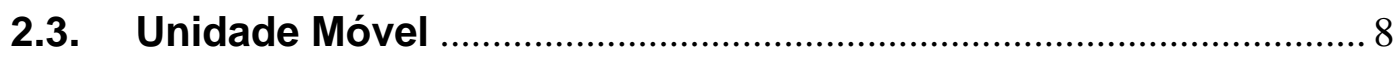

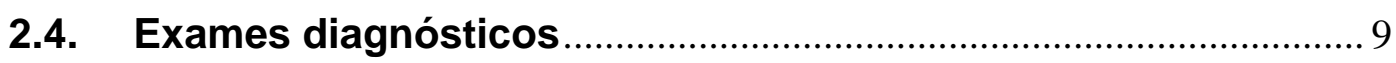

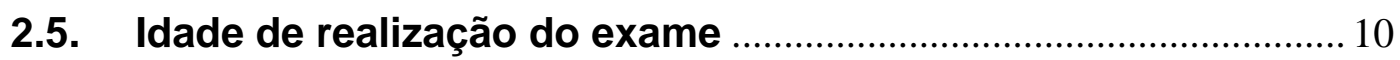

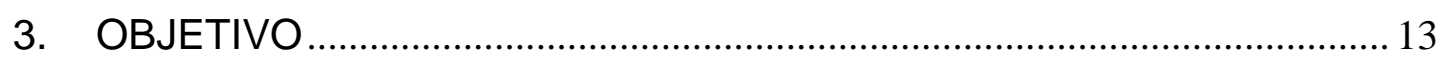

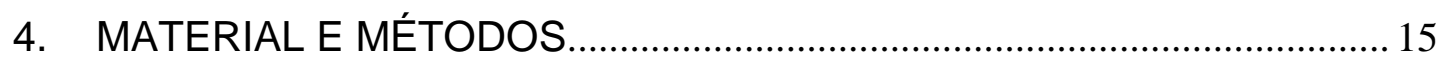

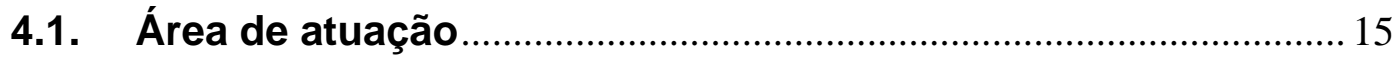

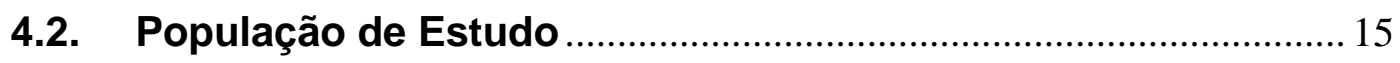

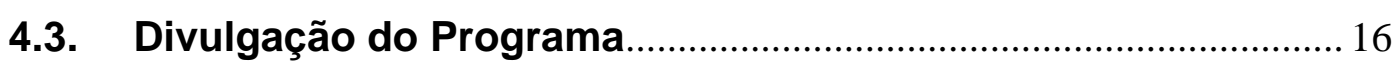

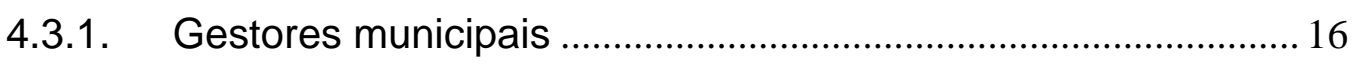

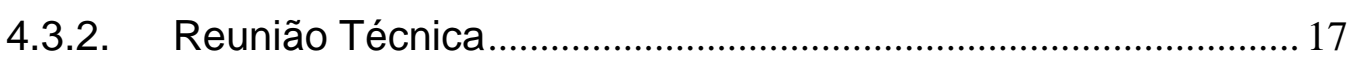

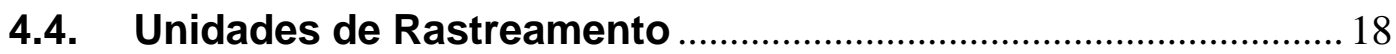

4.4.1. Unidade Móvel (UM) .................................................................. 19

4.4.1.1. Equipe da Unidade Móvel ..................................................... 19

4.4.1.2. Estruturação da Unidade Móvel.............................................20

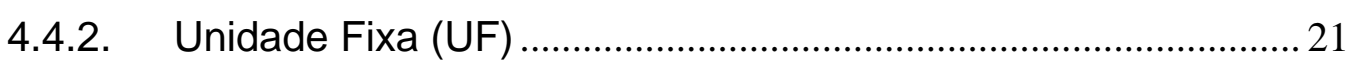

4.4.3. Capacidade Operacional........................................................... 22

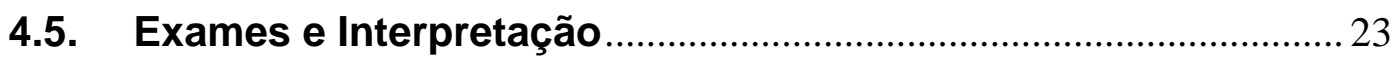

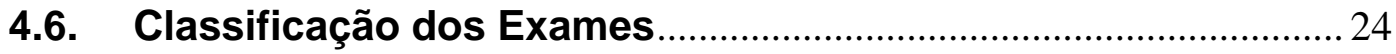

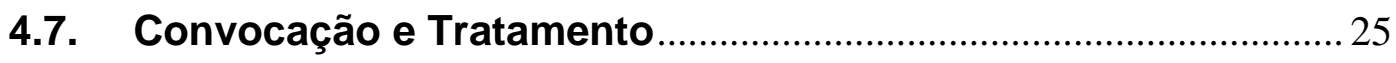

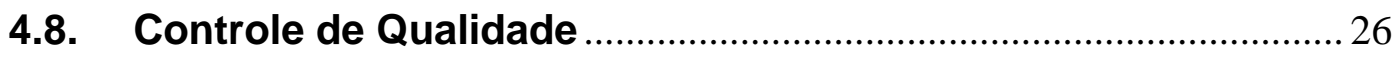

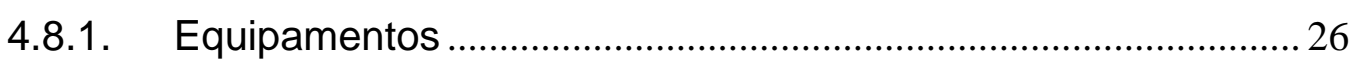

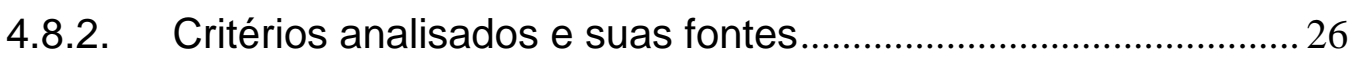

4.8.2.1. European guidelines for quality assurance in breast cancer screening and diagnosis Fourth Edition. 
4.8.2.2. Determinants de La qualité dê programmes organisés de déspsitage du câncer du sein 2003....................................................... 28

4.8.2.3. Sistema de Laudos e Registro de Dados de Imagem da Mama - BI-RADS, 1플 Edição (2005) ...................................................... 28

4.9. Coleta de dados e variáveis de estudo ..........................................29

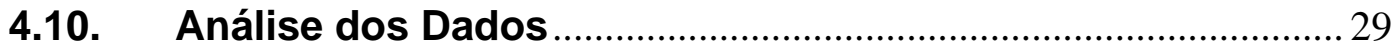

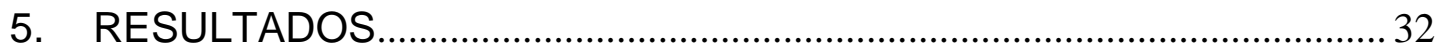

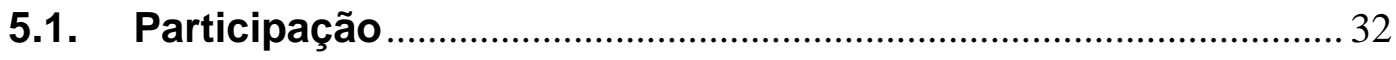

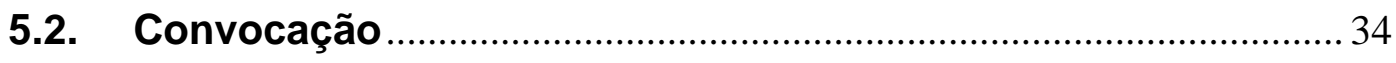

5.3. Confirmação patológica dos casos suspeitos para o câncer de mama 35

5.4. Análise do Sistema de Rastreamento ........................................... 41

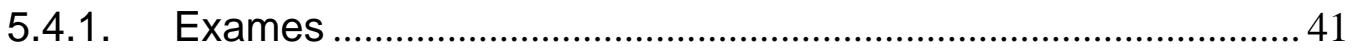

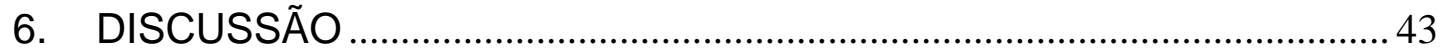

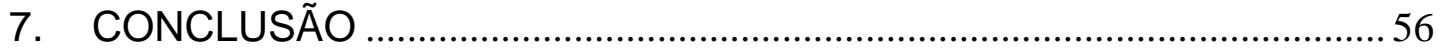

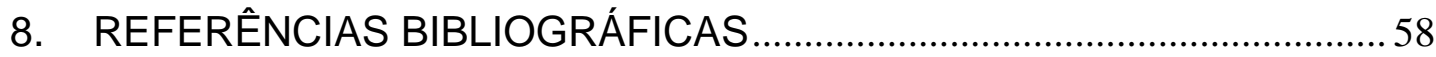
APÊNDICE 



\section{LISTA DE TABELAS}

Tabela 1. Distribuição por faixa etária das mulheres que realizaram exames de mamografia no Sistema de rastreamento do Hospital de Câncer de Barretos em relação ao local de realização do exame.

Tabela 2. Motivos pelos quais as mulheres procuraram atendimento e exames mamográficos.

Tabela 3. Distribuição dos casos de câncer de mama em função de faixa etária e unidade diagnóstica

Tabela 4. Tipos histológicos dos tumores detectados pelo Sistema de rastreamento em cada unidade

Tabela 5. Número de casos de câncer em função do número de mamografias, distribuídas por faixa etária.

Tabela 6. Número de casos de câncer em função do número de mamografias realizadas pela Unidade Móvel, distribuídas por faixa etária.

Tabela 7. Número de casos de câncer em função do número de mamografias realizadas pela Unidade Fixa, distribuídas por faixa etária.

Tabela 8. Comparação entre os resultados do Sistema de Rastreamento com os valores recomendados por órgão internacionais de auditoria em rastreamento mamográficos... 


\section{LISTA DE FIGURAS}

Figura 1. Perfil dos tumores detectados pelo Sistema de Rastreamento Mamográfico, onde UF = Unidade Fixa e UM = Unidade Móvel..................................................... 


\section{RESUMO}

Haikel Jr, RL. Avaliação Inicial de um Programa de Detecção Precoce do Câncer de Mama, por meio de mamografia, na Região de Barretos [tese]. São Paulo: Faculdade de Medicina de São Paulo; 2010.

O câncer de mama é a neoplasia maligna mais prevalente entre as mulheres no mundo e representa $23 \%$ de todos os cânceres femininos. Buscou-se avaliar a implementação de um programa de rastreamento mamográfico para as mulheres que vivem na área de Barretos usando uma unidade móvel (UM) e uma unidade fixa (UF). Um total de 54.238 mulheres com idade entre 40 a 69 anos reside nesta área e são elegíveis para a participação no programa. Os dados epidemiológicos das mulheres foram examinadas entre 01 de abril de 2003 e 31 de março de 2005. A análise estatística foi constituída pela avaliação das frequências dos parâmetros clínicos e as características do tumor usando o teste de Qui-quadrado com correção de Bonferroni, com valor de confiança de $p<0,05$. Um total de 17.964 mulheres (media de 51 anos de idade) foram efetivamente examinadas por mamografia, o que representou $33,1 \%$ de todas as mulheres elegíveis $(18,6$ exames por dia na UF e 26,3 na UM). Setenta e seis casos foram diagnosticados como câncer de mama (41, ou $54 \%$, no UM), o que representa 4,2 casos de câncer de mama para cada 1.000 exames. Foi observada diferença significativa na detecção de câncer entre mulheres com idade entre 50-59 e 60-69 anos $(p<0,001)$ e com idade entre 40-49 e 60-69 anos $(p<0,001)$. Não foram observadas diferenças entre 40 a 49 e 50-59 anos $(p=0,164)$. O programa de rastreamento mamografico é viável no território nacional e os resultados preliminares são animadores.

Descritores: Neoplasia da mama; Programas de rastreamento; Prevenção de câncer de mama; Detecção precoce de câncer; Estágio clínico. 


\section{SUMMARY}

Haikel Jr, RL Initial evaluation of the Breast Cancer Early Detection Program, based on mammography, at Barretos region [thesis]. "São Paulo: Faculdade de Medicina de São Paulo"; 2010.

Breast cancer is the most prevalent malignancy among women worldwide and enrolls $23 \%$ of all female cancers. We sought to evaluate the implementation of a screening program for women who living in Barretos county area using a mobile unit (MU) and a fixed unit (FU). A total of 54,238 women aged 40 to 69 years is living in this area and are eligible for breast screening. Epidemiologic-based data supported the study design and the women were examined from April 01, 2003 to March 31, 2005. Statistical analysis supported the evaluation of clinical parameters frequencies and tumor characteristics using Chi-test and Bonferroni correction test, with confidence value of $p<0,05$. Overall of 17,964 women (media of 51 years old) were effectively examined by mammogram which represented $33,1 \%$ of all eligible women (18,6 in RA and 26,3 exams in MU per day). Seventy-six cases were diagnosed as breast cancer $(41$, or $54 \%$, at $\mathrm{MU})$, which represents 4,2 cases of breast cancer for each 1.000 exams. It was observed significant difference of cancer detection between women aged 50 to 59 and 60 to $69 \mathrm{yrs}(\mathrm{p}<0,001)$ and between women aged 40 to 49 and 60 to $69 \mathrm{yrs}$ $(p<0,001)$. No differences were observed between aged 40 to 49 and 50 to $59 \mathrm{yrs}(p=0,164)$. The program for mammogram screening is feasible to be implementing in Brazil territory and the preliminary results are encouraging.

Descriptors: Breast cancer; Screening programs; Breast cancer prevention; Early detection of cancer; Clinical stage. 


\section{INTRODUÇÃO}

O câncer de mama é a neoplasia mais comum entre as mulheres de todo o mundo e representa $23 \%$ dos diagnósticos de câncer feminino. Suas maiores incidências são observadas no continente Norte Americano, na Austrália e em alguns países europeus. Por outro lado, os países asiáticos e africanos são os que apresentam as menores taxas ${ }^{1}$. A América Latina na sua maioria também apresenta uma baixa taxa de neoplasia mamária, porém a Argentina e o Uruguai apresentam níveis próximos aos europeus, devido a grande imigração daqueles paises ${ }^{2}$.

Segundo o Instituto Nacional de Câncer (INCA) foi esperado para o ano de 2008, um número total de 466.730 casos de câncer no Brasil, sendo 49.400 casos de câncer de mama ${ }^{3}$.

O câncer de mama mostra-se como uma importante patologia que merece atenção dos gestores em saúde pública e leva a uma mobilização para o seu rastreamento e detecção precoce, objetivando com isso a diminuição da sua morbi-mortalidade em nível populacional ${ }^{4}$. 0 rastreamento do câncer tem como premissa a detecção precoce do tumor, antes mesmo que este se torne palpável, o que favorece um tratamento efetivo e, consequentemente, um aumento da sobrevida ${ }^{5}$. A mamografia é o exame de eleição para o rastreamento mamário, sendo considerado o exame de padrão ouro ${ }^{6,7}$ e inúmeros estudos confirmam sua capacidade de detecção precoce do câncer de mama ${ }^{8}$. 
No Brasil, não há de forma efetiva o rastreamento populacional organizado, já que o Ministério da Saúde incita o auto-exame das mamas e realiza mamografia diagnóstica e ultra-sonografia através do Sistema Único de Saúde (SUS). O rastreamento organizado no Brasil, lançado no ano de 2003 pelo INCA, foi implementado unicamente sob a forma de consenso, uma vez que este é apenas um roteiro a ser seguido e não um programa de rastreamento propriamente dito.

A Organização Mundial de Saúde (OMS) estabeleceu como objetivo de um programa de rastreamento a redução da morbi-mortalidade causada pelo câncer, com a utilização de um exame simples de ser realizado, aceito pela população alvo e com um custo aceitável ${ }^{9}$.

A utilização de Unidades Móveis (UMs) tem se mostrado de grande importância no que diz respeito ao acesso de pessoas aos serviços de saúde, como por exemplo, pacientes da terceira idade que usam deste meio para realização de atendimentos ${ }^{10}$. Essa abordagem também tem sido empregada para atendimentos médicos em ambientes de trabalho, onde se consegue estender o atendimento à população sem que as pessoas percam horas de trabalho e gerem prejuízos para as empresas ${ }^{11}$.

As UMs têm sido também empregadas como uma boa estratégia de rastreamento mamográfico, pois com um custo menor, podem atingir um maior número de mulheres. Além do baixo custo essa metodologia tende a facilitar o acesso ao exame de mamografia, já que as UMs levam os mamógrafos a qualquer lugar, e foi evidenciado também um a grande utilização deste meio de rastreamento em pessoas que não possuem meios 
de realizar exame de rastreamento no sistema convencional, seja pela já citada distancia ou mesmo por não estarem amparadas por políticas privadas $^{12,13}$.

Com uma probabilidade de sobrevida de 57\% na população dos países em desenvolvimento e 73\% em países desenvolvidos, o câncer de mama tem sofrido uma intensificação no rastreamento populacional, e essa abordagem elevou para $81 \%$ a taxa de sobrevida em cinco anos das mulheres americanas. ${ }^{14}$. Dados de um estudo espanhol referente ao período de 1975 a 2004 evidenciaram que as mulheres submetidas ao rastreamento apresentaram uma queda de mortalidade da ordem de $62 \%$, contra apenas $22 \%$ de queda naquelas que não participaram. ${ }^{15}$. Apesar de estudos demonstrarem que há diminuição da taxa de mortalidade decorrente dos rastreamentos organizados para o câncer de mama ${ }^{16-18}$, o Brasil efetivamente ainda não possui programa semelhante, o que nos motivou a realização deste rastreamento organizado para o câncer de mama e sua análise neste primeiro ciclo de exames. Uma das perspectivas deste estudo é promover um programa de rastreamento mamográfico organizado na região de Barretos, Estado de São Paulo, obtendo um banco de dados com características sócio-demográficas e histórico de realização do exame mamográfico nas mulheres dos 40 aos 69 anos de idade. 
REVISÃO BIBLIOGRÁFICA 


\section{REVISÃO BIBLIOGRÁFICA}

\subsection{O Câncer de Mama}

O câncer de mama apresenta-se hoje como a neoplasia de maior incidência na população feminina brasileira, e vem apresentando crescimento contínuo em comparação às décadas anteriores ${ }^{9}$. São registrados anualmente 370.000 mortes por esta doença, o que representa $13,9 \%$ das mortes por câncer em mulheres ${ }^{19}$.

No panorama global dos casos de câncer no Brasil o câncer de mama surge em segundo lugar, e na população feminina é a neoplasia maligna de maior incidência. Sua distribuição na população brasileira varia conforme a área geográfica, partindo de 9,74 a 16,81 casos por 100 mil mulheres na região norte do país até 46,77 a 92,77 nas regiões sul e sudeste ${ }^{3}$.

Os países são classificados quanto a sua atitude frente à prevenção do câncer de mama, sendo que o nível básico estimula o auto-exame das mamas, o nível intermediário utiliza-se de mamografia de diagnóstico e ultrasonografia, o nível alto mantém programas de rastreamento, porém sem realizar uma cobertura satisfatória e finalmente o nível máximo que conta com rastreamento populacional organizado ${ }^{20}$. 


\subsection{Rastreamento}

Os primeiros rastreamentos que se tem notícia datam da década de 60 , do século passado ${ }^{21}$. Muitos estudos sobre essa temática, controlados, randomizados e observacionais, têm demonstrado sua eficácia no que diz respeito à redução da morbidade e mortalidade decorrentes do câncer de mama ${ }^{16,18,22,23}$, além de beneficiar a mulher, pois o tratamento torna-se mais ameno e com bons resultados estéticos ${ }^{24}$. Em alguns programas a redução do número de óbitos pode ser de até $39 \%{ }^{17}$.

$\mathrm{Na}$ Holanda o rastreamento mamográfico, com caráter nacional, começou em 1988-89 em dois municípios e se estendeu para várias outras regiões do país depois de alguns anos. Estudo recente com 23.385 mortes por câncer de mama realizado naquele país revelou uma queda de mortalidade de aproximadamente $20 \%$ nas mulheres rastreadas ${ }^{25}$.

No Brasil foi implementado unicamente sob a forma de consenso, lançado no ano de 2003 pelo INCA, lembrando que este é apenas um roteiro a ser seguido e não um programa de rastreamento propriamente dito. Conseqüentemente, esta determinação ocorreu de duas a quatro décadas após o início dos rastreamentos nos países europeus e nos Estados Unidos 26,27

Vários estudos anteriores demonstraram que os países em desenvolvimento apresentam inúmeras dificuldades para a implantação de programas de rastreamento mamográfico, tais como falta de conscientização 
da população e educação dos profissionais de saúde, dificuldade de acesso para diagnóstico e tratamento de câncer, política de rastreamento mal organizada, dificuldades de adesão da população carente aos serviços de mamografias, dificuldade de transporte para a realização de exame na Unidade Fixa e muitos outros ${ }^{28-30}$.

\subsection{Unidade Móvel}

No cenário mundial do rastreamento de câncer de mama a Inglaterra destaca-se pela presença de mais de 90 unidades, com cobertura nacional anual de $75 \%{ }^{31}$.

A UM com mamógrafo tem sido utilizada nos países desenvolvidos para atender subgrupos da população que se submetem menos a exames de rastreamento de câncer de mama. Esses subgrupos são constituídos por mulheres de menor poder aquisitivo, menor escolaridade, idosas, residentes em zona rural, entre outras ${ }^{32-35}$. Essa abordagem também tem sido utilizada no auxílio ao rastreamento organizado, utilizando a UM para visitas a municípios menores, oferecendo acesso fácil e evitando ou reduzindo a locomoção dessa população feminina aos grandes centros ${ }^{36}$.

Interessante relatar que na análise de 82 programas de rastreamento mamográfico realizados na Inglaterra, 86\% destes, utilizam a UM associada à Unidade Fixa ${ }^{37}$. 


\subsection{Exames diagnósticos}

A detecção precoce do câncer de mama pode ser realizada de várias maneiras, que vão desde exames simples como o auto-exame da mama (AEM) até exames complexos de imagem como Ressonância Magnética (RM) da mama.

Por ser simples e não apresentar custos, o auto-exame das mamas é usualmente estimulado pelo corpo clínico, porém a evidência da sua eficácia é muito pequena ${ }^{4,38}$.

O exame clinico das mamas (ECM) não é estudado isoladamente, porém sua associação com a mamografia tem sido alvo de grandes discussões. Um estudo canadense não encontrou nenhuma evidência que defenda a utilização das modalidades diagnósticas associadas, porém nos Estados Unidos esta associação diminuiu a mortalidade por câncer de mama $\operatorname{nos}^{39,40}$.

Apesar de utilizado, uma revisão de literatura internacional concluiu que não há evidências de que o exame ultrassonográfico das mamas seja suficiente em rastreamentos populacionais para o câncer de mama ${ }^{41}$. Outra modalidade de diagnóstico do câncer de mama é a RM mamária. Sua grande contribuição ocorre em populações com riscos elevados para o câncer de mama ${ }^{42}$.

Apesar das novas tecnologias para diagnósticos do câncer de mama, a única aprovada para rastreamento é a mamografia digital. O rastreamento 
mamográfico digital apresentou uma menor taxa de reconvocação, discreta aumento na taxa de detecção de câncer principalmente nas mulheres com mamas densas,os custos ainda mas acreditasse que é questão de tempo para que ocorra a mudança do aparelho analógico para o digital ${ }^{43,44}$. Sua sensibilidade pode variar de $90 \%$ a $94 \%$, dependendo da idade da paciente, sendo que quanto mais idosa a paciente, melhor a sensibilidade do exame 45 .

\subsection{Idade de realização do exame}

A idade recomendada para a paciente ser inserida em um programa de rastreamento mamográfico está entre 40 e 50 anos, sendo que esta variação é tema de numerosos estudos que tentam validar uma faixa etária como ideal para ser o ponto de corte para o início de um rastreamento.

No continente europeu quase a metade dos rastreamentos realizados iniciou suas atividades com mulheres a partir dos 40 anos e mais da metade deles os finalizou com mulheres na faixa dos 69 anos. Esses programas atuam com uma periodicidade bianual do exame mamográfico ${ }^{25}$. A periodicidade vem sendo discutida por muitos autores e Nystrom et al. concluiram que um intervalo superior a doze meses e inferior a trinta e três meses mostrou-se superior ao rastreamento anual ${ }^{46}$, outro estudo mostra que o rsatreamento conduzido na França preconiza um exame a ca dois anos. $^{47}$ 
O Ministério da Saúde, através do documento de consenso para o rastreamento do câncer de mama, redigido pelo INCA, preconiza que o início do rastreamento com exames mamográficos no Brasil deva dar-se a partir dos 50 anos de idade, devendo ser repetidos em até dois anos e finalizá-los com mulheres de 69 anos. Entretanto, a lei brasileira 11.664 de 29/04/2008 sugere o inicio do rastreamento mamográfico em mulheres de 40 anos. 


\section{OBJETIVO}

Tem-se como objetivo maior avaliar a implantação e os resultados obtidos no primeiro turno do rastreamento mamográfico em mulheres residentes no Diretório Regional de Saúde V que, espontaneamente buscaram atendimento nas Unidades participantes. 
MATERIAL E MÉTODOS 


\section{MATERIAL E MÉTODOS}

\section{1. Área de atuação}

O local escolhido para a realização do projeto foi a região com denominação de Diretório Regional de Saúde V (DRS V), segundo a Secretaria de Saúde do Estado de São Paulo. Este Diretório conta com dezenove municípios: Altair, Barretos, Bebedouro, Cajobi, Colina, Colômbia, Embaúba, Guaíra, Guaraci, Jaborandi, Monte Azul Paulista, Olímpia, Severínia, Taiaçú, Taiúva, Taquaral, Terra Roxa, Viradouro, Vista Alegra do Alto.

A DRS V apresenta uma área territorial de 8.182 km2 e 406.885 habitantes, segundo dados do Instituto Brasileiro de Geografia e Estatística (IBGE, 2005), e sua sede está localizada no município de Barretos (20³3'26" Sul, 48³4'04" Oeste), Estado de São Paulo.

\subsection{População de Estudo}

O rastreamento do câncer de mama estabeleceu que a população alvo fosse de mulheres com idade entre 40 e 69 anos e residentes em uma das quaisquer cidades da DRS V, com isso obtivemos, segundo dados do 
IBGE, uma população elegível de 54.238 mulheres, sendo 15.500 do município de Barretos e 38.738 das demais cidades dessa DRS.

As mulheres que realizaram mamografia há menos de dois anos e as com história pregressa de câncer de mama foram excluídas, também não fizeram parte dos dados mamografias de mulheres que realizaram mais de um exame no período, lembrando que o considerado foi sempre o primeiro exame realizado.

O estudo é baseado em dados epidemiológicos colhidos no período de 01 de abril de 2003 até a data de 31 de março de 2005 e dados clínicos observados retrospectivamente.

\subsection{Divulgação do Programa}

\subsubsection{Gestores municipais}

Nas cidades participantes foi realizada uma reunião para a explanação do projeto junto aos gestores, representados pelo prefeito e secretários de saúde. Esta reunião foi marcada após contato telefônico com o município e a visita agendada conforme a disponibilidade, não devendo a data superar duas semanas após primeiro contato.

Nesta reunião foram abordadas as linhas gerais do programa e sua relação com o Sistema Único de Saúde (SUS), e os gestores foram informados que não teriam custos adicionais e nem cotas mínimas ou 
máximas de realização de exames. Ficou a cargo do município apenas a mobilização das mulheres, o apoio logístico para realização do exame e o transporte deste paciente até a Fundação PIO XII, para possíveis exames complementares e tratamentos, situação esta que já ocorre, visto que a Instituição é referência na região para tratamento de pacientes com câncer.

Os gestores também indicaram profissionais da área de saúde para o treinamento da parte técnica do programa. Na sua grande maioria estes profissionais faziam parte do Programa de Saúde da Família (PSF), dado o fato de apresentarem maior contato com a população, através de visitas domiciliares, como preconizam as diretrizes do PSF.

\subsubsection{Reunião Técnica}

A reunião com os profissionais indicados pelos gestores deu-se no município de origem, onde foi realizada a explanação sobre o sistema de rastreamento, suas diretrizes e seus objetivos. Ao término de cada reunião foi apresentado a estes profissionais os impressos que deveriam ser preenchidos pelo município.

Nesta primeira reunião ficou estipulado o calendário para o primeiro trimestre do projeto, tempo aproximado que a Unidade Móvel (UM) levaria para percorrer os 18 municípios. Por um motivo logístico e de organização, o município sede não foi atendido pela UM, ficando a cargo somente da Unidade Fixa (UF). 
A divulgação do programa ficou a cargo dos municípios, conforme orientação, porém não foi imposto pelo Hospital de Câncer qual o método que deveria ser utilizado. As enfermeiras dos municípios também foram orientadas quanto ao fluxo dos exames, classificados como exames normais, onde as mulheres deveriam repetir o exame em dois anos e, exames alterados, que deveriam passar em consulta na Instituição. Essas enfermeiras receberam também orientações de como abordar as pacientes, principalmente aquelas que apresentavam exames alterados.

Determinou-se também que ao final de cada ciclo trimestral, realizaríamos uma reunião na Fundação PIO XII, onde seriam discutidos planos futuros, dificuldades, resultados e o calendário do próximo trimestre.

A primeira cidade do trimestre seguinte seria avisada um mês antes da reunião, pois este período foi estipulado como ideal para a captação de mulheres nos municípios.

Os profissionais do município deveriam preencher a ficha cadastral (Anexo I) antes da data do atendimento e os demais formulários seriam preenchidos no momento do exame.

\subsection{Unidades de Rastreamento}

As unidades de rastreamento foram dividas em Unidade Móvel (UM) e Unidade Fixa (UF). 


\subsubsection{Unidade Móvel (UM)}

A UM percorreu os dezoito municípios da DRS V, permanecendo de dois a cinco dias em cada município. Este período foi determinado de acordo com o número de habitantes de cada município, porém, sem critérios rígidos de distribuição.

Foram disponibilizadas 40 vagas por dia na UM, divididas em dois períodos. O agendamento das mulheres foi realizado com intervalos de 15 minutos, conforme orientações. No caso de algum município apresentar um número de mulheres superior ao estipulado, seriam atendidas da mesma forma.

O período de atendimento foi pré-determinado, em horário entre 08:00 e 17:00 horas, podendo estender-se ou ser alterado segundo solicitação prévia do município.

\subsubsection{Equipe da Unidade Móvel}

A UM possui uma equipe formada por:

- Motorista

- Enfermeira

- Duas Técnicas de Radiologia 
- Técnico em Câmara Escura

\subsubsection{Estruturação da Unidade Móvel}

A UM foi desenvolvida no ano de 2002 através de uma parceria da Fundação Pio XII e o Escritório de Engenharia Karmam e Associados, com sede na cidade de São Paulo. A Unidade foi construída num chassi da montadora Mercedes Bens, com a especificação OF 1418-52, sob o qual foi montada uma estrutura própria. Esta estrutura era bastante semelhante a um ônibus tradicional, porém, não possuía janelas ou bancos, mantendo as portas dianteira e traseira. (Anexo III)

A estruturação interna da Unidade ficou a cargo da empresa Revescap, especializada no desenvolvimento e montagem de Unidades Móveis (Anexo II). Esta unidade é também composta por duas salas ginecológicas para a coleta de exame citológico (Papanicolaou), mas não serão consideradas no momento, por não fazerem parte deste estudo.

Apesar de não existir legislação referente à utilização de UM para a realização de exames radiológicos, todas as resoluções instituídas para o devido funcionamento de unidades diagnósticas foram seguidas, respeitando a Resolução SS-625/94 e a Portaria MS/SVS 453/98.

A UM é equipada com um mamógrafo da marca General Eletric $\AA$ (GE), modelo Senography 700 T (GE - França - Fevereiro 2002), que foi instalado seguindo as orientações técnicas do setor responsável da GE (Site 
Planning). Esta unidade possui também uma câmara escura equipada com uma processadora, modelo MIN R Mamography Kodak®, (México 2003) regulada para o processamento de filmes mamográficos. Os filmes utilizados eram da marca Kodak® EV Film e os chassis da mesma empresa. Todos os possíveis produtos agressores do meio ambiente que resultam do processamento de películas radiográficas tiveram o seu descarte feito conforme a legislação vigente.

A UM possui um sistema de informação, que se caracteriza por um banco de dados que poderia ser consultado em um computador portátil. Este banco era atualizado semanalmente na Fundação PIO XII para impedir a realização de exames repetidos em período inferior a dois anos e constava basicamente por uma planilha elaborada em Software Microsoft Excel $\AA_{\text {, }}$ onde os dados cadastrais das mulheres eram digitados.

\subsubsection{Unidade Fixa (UF)}

Denominou-se Unidade Fixa (UF) o Departamento de Diagnóstico por Imagem do Hospital de Câncer de Barretos. Essa UF, conta com três mamógrafos da marca General Eletric $\circledast$, sendo um modelo Senography 600 T (GE - França - Junho 1993), um Senography 700 T (GE - França - Abril 2002) e o terceiro modelo, um Senography MDR (GE - França - Junho 2002). A UF possui ainda uma processadora da marca KODAK, modelo MIN-R Mamography (México 2003) e os demais itens, como filmes e chassis, são semelhantes aos utilizados na UM. 
O número de vagas nessa Unidade era de 120 exames por dia, sendo que as mulheres deveriam ser encaminhadas pelas enfermeiras dos municípios após contato prévio com o Departamento de Prevenção. Esse cuidado foi tomado para que não houvesse problemas de fluxo no Departamento de Radiologia. Essas mulheres já possuíam uma ficha cadastral previamente preenchida e no momento do exame esse cadastro era digitado no banco de dados para evitar duplicidade de exames.

Devemos observar que a unidade móvel não realizou exames no município de Barretos, ficando esta população apenas com acesso à unidade fixa, diferentemente das outras 18 cidades que além da unidade móvel também tiveram acesso a fixa, porem as formas de divulgação e convocação que foram utilizadas em Barretos foram as mesmas das outras cidades participantes.

\subsubsection{Capacidade Operacional}

A capacidade de atendimento das duas unidades foi de 64.000 exames, número suficiente para a cobertura de toda a população elegível.

O numero final computado como capacidade operacional, foi um produto da multiplicação do numero de exames diários (40), do numero de dias úteis por mês(20 em media), por dez meses de utilização dos equipamentos(não foi utilizados doze meses pois devemos observar manutenções preventivas, possíveis quebras ou outro pressuposto que 
possa surgir) e finalmente por dois que é o tempo estabelecido para a repetição do exame.

A meta do rastreamento era de $70 \%$ da população elegível, com isso 37.967 exames deveriam ser realizados nos dois anos do primeiro turno.

\subsection{Exames e Interpretação}

Os exames de mamografias foram realizadas em duas incidências (crânio-caudal e médio-lateral), foram interpretados através de leitura simples, na Fundação PIO XII, que conta com nove médicos radiologistas com experiência comprovada na interpretação de exames mamográficos, esse corpo clinico conta com titulação junto ao Colégio Brasileiro de Radiologia, mesma instituição que outorgou para o Departamento o Certificado de Qualidade em Mamografia, desde o ano de 1997.

O Departamento de Imagem realiza cerca de 2.100 exames de mamografia por mês, esses exames são divididos entre os profissionais, cabendo a cada um deles uma média de 3.150 exames por ano. Neste estudo não foi realizado a dupla leitura dos exames mamográficos. 


\subsection{Classificação dos Exames}

Os exames foram classificados segundo a Primeira Edição Brasileira do BI-RADS (2005), baseada na quarta edição americana. A classificação utilizada segue abaixo:

- Categoria 0: Avaliação mamográfica incompleta, necessita avaliação adicional;

- Categoria 1: Negativa, Avaliação mamográfica completa;

- Categoria 2: Achados benignos, porém com descrição de alguma alteração;

- Categoria 3: Provavelmente benigno, porém necessita de exames de seguimento;

- Categoria 4: Anormalidade suspeita; sugere-se biópsia da área;

- Categoria 5: Altamente sugestivo de malignidade, necessário Avaliação Histológica;

A categoria 3 foi excluída do exame inicial, pois segundo BI-RADS é aconselhável uma completa avaliação antes de se confirmar uma avaliação como provavelmente benigna, portanto no primeiro exame esta classificação foi suprimida em favor da categoria 0 . 


\subsection{Convocação e Tratamento}

Os laudos emitidos pelos médicos radiologistas foram enviados ao Departamento de Prevenção do Hospital de Câncer para triagem e convocações. Os exames categorizados como BI-RADS 1 e 2 foram considerados normais e seguiram para as enfermeiras dos seus respectivos municípios. As mulheres que apresentaram mamografias com categorias 0 , 4 e 5 foram convocadas por intermédio do Departamento de Prevenção. Esse mesmo Departamento encaminhou mensagem via FAX para a enfermeira do município, com os nomes e datas das consultas das pacientes. Caso a paciente não comparecesse à consulta, novo contato era realizado com a enfermeira, e em casos de reincidência, o contato era feito diretamente com a paciente.

As consultas foram realizadas no ambulatório da Fundação PIO XII, por médicos cirurgiões oncologistas, que elaboraram história clinica em ficha própria (Anexo IV).

Os exames complementares, como biópsias e demais procedimentos, foram solicitados conforme a padronização do Departamento de Mastologia. Os casos diagnosticados como câncer de mama foram tratados na Instituição em toda sua plenitude.

Nos casos em que o resultado anatomopatológico da biópsia mostrouse negativo, ou qualquer outro exame mamográfico de seguimento onde se evidenciasse a classificação 3, a proposta seria um seguimento por 24 
meses, realizando exame de mamografia nas datas de seis, doze e vinte e quatro meses contados a partir do primeiro exame. Ao término desse período, caso o exame não apresentasse alteração, a paciente seria liberada para retornar ao rastreamento.

\subsection{Controle de Qualidade}

\subsubsection{Equipamentos}

O controle de qualidade dos equipamentos, tanto do mamógrafo quanto da processadora, foi realizado segundo o que preconiza a Agência Nacional de Vigilância Sanitária (ANVISA).

As duas Unidades passaram por testes de qualidade periódicos. Os testes de processamento, com a utilização de sensitômetro e densitômetro foram realizados diariamente e os de imagem, com a utilização de um Phanton foram conduzidos uma vez por semana.

\subsubsection{Critérios analisados e suas fontes}

O controle de qualidade do rastreamento foi feito buscando avaliar qualidade segundo as normas do rastreamento e foi baseado em alguns critérios que fazem parte da primeira versão em português do Sistema de Laudos e Registro de Dados de Imagem da Mama - BI-RADS, da quarta 
versão do European guidelines for quality assurance in breast cancer screening and diagnosis e do Determinants de la qualité dês programmes organisés des déspistage du câncer du sein, Canada de 2003. Esses critérios estão descritos abaixo:

\subsubsection{European guidelines for quality assurance in breast cancer screening and diagnosis Fourth Edition}

- Taxa de participação - número de mulheres que participaram efetivamente do rastreamento, em relação àquelas elegíveis para o programa de rastreamento.

- Taxa de reconvocação - número de mulheres que foram reconvocadas para exames adicionais em relação ao número de participantes do rastreamento.

- Taxa de detecção de câncer - número de diagnósticos positivos para câncer em relação a 1000 mulheres rastreadas.

- Proporção de casos de câncer invasivo em relação ao número total de cânceres detectados.

- Proporção de casos com estadiamento II em relação ao total de cânceres detectados.

- Proporção dos casos invasivos com linfonodo negativo.

- Proporção dos casos positivos com tumor menor que $10 \mathrm{~mm}$. 


\subsubsection{Determinants de La qualité dê programmes} organisés de déspsitage du câncer du sein 2003

- Taxa de detecção do câncer invasivo - número de mulheres que foram detectadas com carcinoma invasivo da mama em relação a 1000 mulheres examinadas.

\subsubsection{Sistema de Laudos e Registro de Dados de Imagem da Mama - BI-RADS, 1ㄹ Edição (2005)}

- Linfonodo axilar positivo - número de pacientes que possuíam comprometimento ganglionar axilar.

- Valor Preditivo Positivo 1 - determina quão freqüente os exames alterados são diagnosticados como cancer.

- Valor Preditivo Positivo 2 - determina a indicação correta da biopsia, quantas são positivas.

- Estadiamento 0 e I - número de pacientes que se encontravam classificadas nas categorias 0 e I segundo a Classificação de Tumores Malignos (TNM) da União Internacional Contra o Câncer (UICC)

Os critérios de qualidade que se encontravam repetidos e com os mesmos valores de referencia foram considerados apenas uma vez. 


\subsection{Coleta de dados e variáveis de estudo}

A coleta das informações das pacientes, referentes aos exames mamográficos foi realizada pelas enfermeiras das Unidades através de entrevistas. Para as mulheres convocadas para nova avaliação foi criado um Registro Geral Hospitalar (RGH) e aberto prontuário médico, depositado e catalogado no Serviço de Arquivo Médico e Estatística (SAME) do Hospital de Câncer de Barretos. Desses prontuários foram coletadas informações de seguimento, como os resultados dos novos exames radiológicos e das biópsias, por exemplo.

As variáveis estudadas foram sócio-demográficas (sexo e idade), clínicas (sintomas e alterações mamárias), radiológicas (mamografia), anatomopatológicas (tipo histológico, tamanho do tumor, comprometimento das cadeias linfonodais e estádio) e meio pelo qual as mulheres foram convocadas para o exame.

Em virtude de ser um estudo de rastreamento, os Comitês de Ética em Pesquisa (CEP) das instituições não solicitaram a aplicação do termo de consentimento livre e esclarecido (TCLE) para estas pacientes.

\subsection{Análise dos Dados}

A base de dados das mulheres que participaram do Sistema de Rastreamento foi montada em planilha do Software Microsoft Office Excel® 
e posteriormente exportadas para o Software SPSS for Windows® v. 16.0., onde foram efetuadas as análises estatísticas.

A análise estatística consistiu da descrição das freqüências das variáveis, calculando-se os percentuais para cada categoria, bem como a mediana para o tamanho do tumor na macroscopia. As significâncias das diferenças encontradas entre as categorias foram acessadas através do teste de associação pelo qui-quadrado. Para as comparações múltiplas foi realizada a Correção de Bonferroni, onde o valor de a é dividido pelo número de comparações.

Foi adotado valor de significância $\mathrm{p}<0,05$. 
RESULTADOS 


\section{RESULTADOS}

\subsection{Participação}

A população elegível para o programa foi de 54.238 mulheres e destas, 17.964 realizaram exames mamográficos, com uma taxa de participação de 33,1\%. Neste período foram realizados 20.897 exames e excluídos 2.933, pois se tratavam de exames repetidos indevidamente, ou seja, foram realizados em periodicidade inadequada, antes do prazo de dois anos. O local de maior procura para a realização do exame de mamografia foi a Unidade Móvel, que realizou 10.521 exames, o que corresponde a $58,6 \%$ das mulheres. A UM foi o local também onde foi notada a maior taxa de exames repetidos indevidamente, com $69 \%$ do total.

As unidades do rastreamento realizaram por dia em média 18,6 exames na UF e 26,3 exames na UM. A idade média e mediana das mulheres que aderiram ao programa foi de 51 e 50 anos, respectivamente. A faixa etária predominante foi a de 40 a 49 anos (48,2\%), seguida pela faixa etária de 50 a 59 anos (34,4\%) e finalmente 60 a 69 anos $(17,4 \%)$ nas duas unidades de exames (Tabela 1). 
Tabela 1. Distribuição por faixa etária das mulheres que realizaram exames de mamografia no Sistema de rastreamento do Hospital de Câncer de Barretos em relação ao local de realização do exame.

\begin{tabular}{ccccc}
\hline \multirow{2}{*}{ Local exame } & \multicolumn{4}{c}{ Idade (anos) } \\
\cline { 2 - 5 } & $\mathbf{4 0 - 4 9}$ & $\mathbf{5 0 - 5 9}$ & $\mathbf{6 0 - 6 9}$ & Total \\
\hline Unidade Fixa & $3.642(48,9)$ & $2.613(35,1)$ & $1.188(16,0)$ & $7.443(100,0)$ \\
Unidade Móvel & $5.024(47,9)$ & $3.560(33,8)$ & $1.937(18,4)$ & $10.521(100,0)$ \\
\hline Total & $8.666(48,2)$ & $6.173(34,4)$ & $3.125(17,4)$ & $17.964(100,0)$
\end{tabular}

Do total de participantes do programa, $42,1 \%$ (7.560) nunca haviam feito mamografia e $57,9 \%$ (10.404) realizaram o exame em algum momento da vida. Dentre os diversos motivos ou meios de convocação para a realização da mamografia de que o programa dispunha o que se mostrou de melhor efeito e impacto foi a atuação do agente comunitário, responsável por $46,8 \%$ das mulheres convocadas. O encaminhamento médico respondeu por $27,1 \%$, a mídia foi responsável pela convocação de $12,4 \%$ das mulheres, a conversa com vizinhos com $5,7 \%$ e finalmente outros motivos com $8,1 \%$. (Tabela 2). 
Tabela 2. Motivos pelos quais as mulheres procuraram atendimento e exames mamográficos.

\begin{tabular}{cccc}
\hline & \multicolumn{3}{c}{ Local exame } \\
Motivo da & Unidade Fixa & Unidade Móvel & Total \\
Procura & $\mathbf{n}(\%)$ & $\mathbf{n}(\%)$ & $\mathbf{n}(\%)$ \\
\hline CV & $221(3,0)$ & $805(7,7)$ & $1.026(5,7)$ \\
EM & $3.886(52,2)$ & $975(9,3)$ & $4.861(27,1)$ \\
Mídia & $466(6,3)$ & $1.753(16,7)$ & $2.219(12,4)$ \\
PSF & $2.317(31,1)$ & $6.089(57,9)$ & $8.046(46,8)$ \\
Outro & $553(7,4)$ & $899(8,5)$ & $1.452(8,1)$ \\
\hline Total & $7.443(100,0)$ & $10.521(100,0)$ & $17.964(100,0)$ \\
\hline
\end{tabular}

Onde: $\mathrm{CV}=$ conversa com vizinhos; $\mathrm{EM}=$ encaminhamento médico; PSF = Programa de Saúde da Família.

\subsection{Convocação}

As mulheres que apresentaram alguma alteração na mama, detectada pelos exames mamográficos, foram reconvocadas para exames adicionais. Dentre as 17.964 mulheres examinadas, 1.690 apresentaram essas características e, dessa forma, a taxa de reconvocação foi de $9,4 \%$ (1690/17.964).

Após avaliação clínico-radiológica adequada, 2,63\% (474/17.964) das pacientes reconvocadas foram encaminhadas para o Departamento de Radiologia para realização de biópsia diagnóstica, onde 0,8\% (143/17.964) das pacientes foram submetidas a core biopsy guiado por mamografia ou ultra sonografia. As demais 1,83\% (331/17.964) foram submetidas à biópsia 
a céu aberto, guiadas por fio ou mesmo guiadas por Radioguided occult lesion localisation (ROLL).

\subsection{Confirmação patológica dos casos suspeitos para o câncer de mama}

Após procedimento diagnóstico foram confirmados 76 casos de câncer de mama. A detecção mostrou-se ligeiramente maior na Unidade Móvel, que realizou 53,9\% (41 casos) dos diagnósticos contra 46,1\% da Unidade Fixa (35 casos). A idade média das mulheres, no momento da confirmação, foi de 52 anos (41-68 anos), a mediana foi de 50 anos e a faixa etária predominante foi a de 40 a 49 anos, com $34(44,7 \%)$ casos confirmados (Tabela 3).

O valor preditivo positivo 1 (VPP1) que determina quantos exames alterados foram câncer foi de 4,49\% e o valor preditivo positivo 2 (VPP2) que determina a indicação precisas da biopsia foi de $16 \%$.

As análises das informações referentes à realização prévia de exames de mamografia nas mulheres que apresentaram resultado positivo para câncer de mama evidenciaram três situações: presença de mamografia prévia, realizada em dois anos que foi denominada prevenção adequada (PA), mulheres que realizaram mamografia há mais de dois anos, denominada prevenção inadequada $(\mathrm{PI})$ e finalmente mulheres que nunca realizaram o exame 
Tabela 3. Distribuição dos casos de câncer de mama em função de faixa etária e unidade diagnóstica.

\begin{tabular}{lccc}
\hline \multicolumn{1}{c}{ Faixa etária } & UM & UF & Total \\
& $\mathbf{n}(\%)$ & $\mathbf{n}(\%)$ & $\mathbf{n}(\%)$ \\
\hline $40-49$ anos & $20(58,8)$ & $14(42,2)$ & $34(44,7)$ \\
$50-59$ anos & $8(38,1)$ & $13(61,9)$ & $21(27,6)$ \\
$60-69$ anos & $13(61,9)$ & $8(38,1)$ & $21(27,6)$ \\
\hline Total & $41(53,9)$ & $35(46,1)$ & $76(100,0)$ \\
\hline
\end{tabular}

Onde: UM = Unidade Móvel; UF = Unidade Fixa

Observamos que $31,5 \%(24 / 76)$ das mulheres realizavam a prevenção adequadamente, sendo que as que realizaram o exame na UM somaram $24,4 \%$ (10/41), e na UF o resultado foi $40,0 \%$ (14/35). As mulheres que realizaram mamografia pelo menos uma vez na vida, porém a mais de dois anos, foi de $9,2 \%$ (7/76) divididos da seguinte forma: 5,7\% (2/35) foram observadas na UF contra $12,2 \%(5 / 41)$ na UM. A maioria das mulheres nunca realizou o exame e correspondiam a $36,8 \%(28 / 76)$ do total, sendo que $40,0 \%$ (14/35) delas foram rastreadas pela UF e $31,7 \%(13 / 41)$ na UM. Não obtivemos os dados de 22,3\% (17/76) das mulheres com diagnóstico positivo.

Os laudos anatomopatológicos das biópsias confirmaram uma prevalência de carcinomas do tipo ductal invasivo $(72,4 \%)$, seguido por lobular invasivo $(17,1 \%)$ e ductal in situ $(10,5 \%)$. Os tipos histológicos e suas freqüências estão dispostos na Tabela 4. A comparação dos tipos 
histológicos entre as unidades de rastreamento mostrou uma diferença estatisticamente significativa na detecção de carcinomas in situ pela UF em relação a UM $(p<0,001)$.

Tabela 4. Tipos histológicos dos tumores detectados pelo Sistema de rastreamento em cada unidade.

\begin{tabular}{lccc}
\hline Tipo Histológico & UF & UM & Total \\
& $\mathbf{n}(\%)$ & $\mathbf{n}(\%)$ & $\mathbf{n}(\%)$ \\
\hline Ductal in situ & $5(14,3)$ & $3(7,3)$ & $8(10,5)$ \\
Ductal invasivo & $22(62,9)$ & $33(80,5)$ & $55(72,4)$ \\
Lobular invasivo & $8(22,8)$ & $5(12,2)$ & $13(17,1)$ \\
\hline
\end{tabular}

Onde: UM = Unidade Móvel; UF = Unidade Fixa

Levando-se em conta o número de mamografias realizadas, obtevese uma taxa de detecção global de 4,2 casos de câncer por 1.000 exames. Esta taxa mostrou-se maior na população mais idosa (60 a 69 anos), seguida pela faixa etária de 40 a 49 anos e posteriormente pelas mulheres entre 50 e 59 anos $(p<0,001)$ (Tabela 5). A taxa de detecção por 1000 exames mostrou-se significativamente maior na Unidade Fixa, com 4,7/1.000 exames contra 3,9/1.000 na Unidade Móvel $(p<0,001)$ (Tabelas 6 e 7). 
Tabela 5. Número de casos de câncer em função do número de mamografias, distribuídas por faixa etária.

\begin{tabular}{cccc}
\hline Faixa etária & Mamografias & Casos de câncer & $\begin{array}{c}\text { № câncer / } \\
\mathbf{1 . 0 0 0 ~ M M G ~}\end{array}$ \\
\hline 40 a 49 anos & $8.666(48,2 \%)$ & $34(44,7 \%)$ & 3,92 \\
50 a 59 anos & $6.173(34,4 \%)$ & $21(27,6 \%)$ & 3,40 \\
60 a 69 anos & $3.125(17,4 \%)$ & $21(27,6 \%)$ & 6,72 \\
\hline Total & $17.964(100,0 \%)$ & $76(100,0 \%)$ & 4,23 \\
\hline Onde: $\mathrm{MMG}=$ mamografia
\end{tabular}

Tabela 6. Número de casos de câncer em função do número de mamografias realizadas pela Unidade Móvel, distribuídas por faixa etária.

\begin{tabular}{cccc}
\hline \multirow{2}{*}{ Faixa etária } & $\begin{array}{c}\text { Mamografias/ } \\
\text { Unidade Móvel }\end{array}$ & Casos de câncer & $\begin{array}{c}\text { No câncer / } \\
\mathbf{1 . 0 0 0 ~ M M G ~}\end{array}$ \\
\hline 40 a 49 anos & $5.024(47,8 \%)$ & $20(48,7 \%)$ & 3,98 \\
50 a 59 anos & $3.560(33,8 \%)$ & $8(19,5 \%)$ & 2,24 \\
60 a 69 anos & $1.937(18,4 \%)$ & $13(31,8 \%)$ & 6,71 \\
\hline Total & $10.521(100,0 \%)$ & $41(100,0 \%)$ & 3,89 \\
\hline
\end{tabular}

Onde: $\mathrm{MMG}=$ mamografia

Quando analisada a taxa de detecção por faixa etária constatou-se uma diferença estatisticamente significativa entre as mulheres de 50 a 59 e 60 a 69 anos $(p<0,001)$ e entre as mulheres de 40 a 49 e 60 a 69 anos $(p<0,001)$. Não encontramos diferença estatisticamente significativa entre as mulheres de 40 a 49 e 50 a 59 anos $(p=0,164)$. 
Tabela 7. Número de casos de câncer em função do número de mamografias realizadas pela Unidade Fixa, distribuídas por faixa etária.

\begin{tabular}{cccc}
\hline Faixa etária & $\begin{array}{c}\text { Mamografias/ } \\
\text { Unidade Fixa }\end{array}$ & Casos de câncer & № câncer / \\
& & & 1.000 MMG \\
\hline 40 a 49 anos & $3.642(48,9 \%)$ & $14(40 \%)$ & 3,84 \\
50 a 59 anos & $2.613(35,1 \%)$ & $13(37,1 \%)$ & 4,97 \\
60 a 69 anos & $1.188(16,0 \%)$ & $8(22,8 \%)$ & 6,73 \\
\hline Total & $7.443(100,0 \%)$ & $35(100,0 \%)$ & 4,70
\end{tabular}

Onde: $\mathrm{MMG}=$ mamografia

Depois de ressecadas as lesões mostraram um tamanho mediano de $1,6 \mathrm{~cm}(0,1-8,0 \mathrm{~cm})$ pela macroscopia. Dentre todos os tumores, $36(47,3 \%)$ tinham menos de um centímetro e meio $(1,5 \mathrm{~cm})$, em seu maior eixo e dos casos estadiados como EC II, nove $(11,8 \%)$ apresentavam-se como tumores menores que dois centímetros. Já a microscopia revelou que 39 casos $(57,4 \%)$ dos carcinomas infiltrantes não apresentavam linfonodos axilares comprometidos por células malignas.

O estadiamento dos tumores, segundo a Classificação de Tumores Malignos (TNM) da União Internacional Contra o Câncer (UICC), mostrou que $43,4 \%$ do total de casos diagnosticados se apresentaram em estádios iniciais de desenvolvimento (EC 0 e EC I), sendo 48,6\% deles detectados na UF e $39,1 \%$ na UM (Figura 1). Os estadiamentos avançados (EC III e EC IV) representaram $15,8 \%$ de todos os tumores, sendo que $14,3 \%$ dos tumores mais avançados foram detectados na UF e 17\% na UM (Figura 1). Partindo 
do pressuposto de que não há diferença no estadiamento entre as Unidades Fixa e Móvel, a probabilidade dessa diferença ser explicada ao acaso é de $0,3 \%$. Dessa forma constatou-se que há diferença estatisticamente significativa no estadiamento entre as Unidades $(p=0,003)$.

Os casos de pacientes com estadiamento II correspondeu a $40,8 \%$, onde $43,9 \%$ foram rastreados pela Unidade Móvel e 37,1\% pela Unidade Fixa.

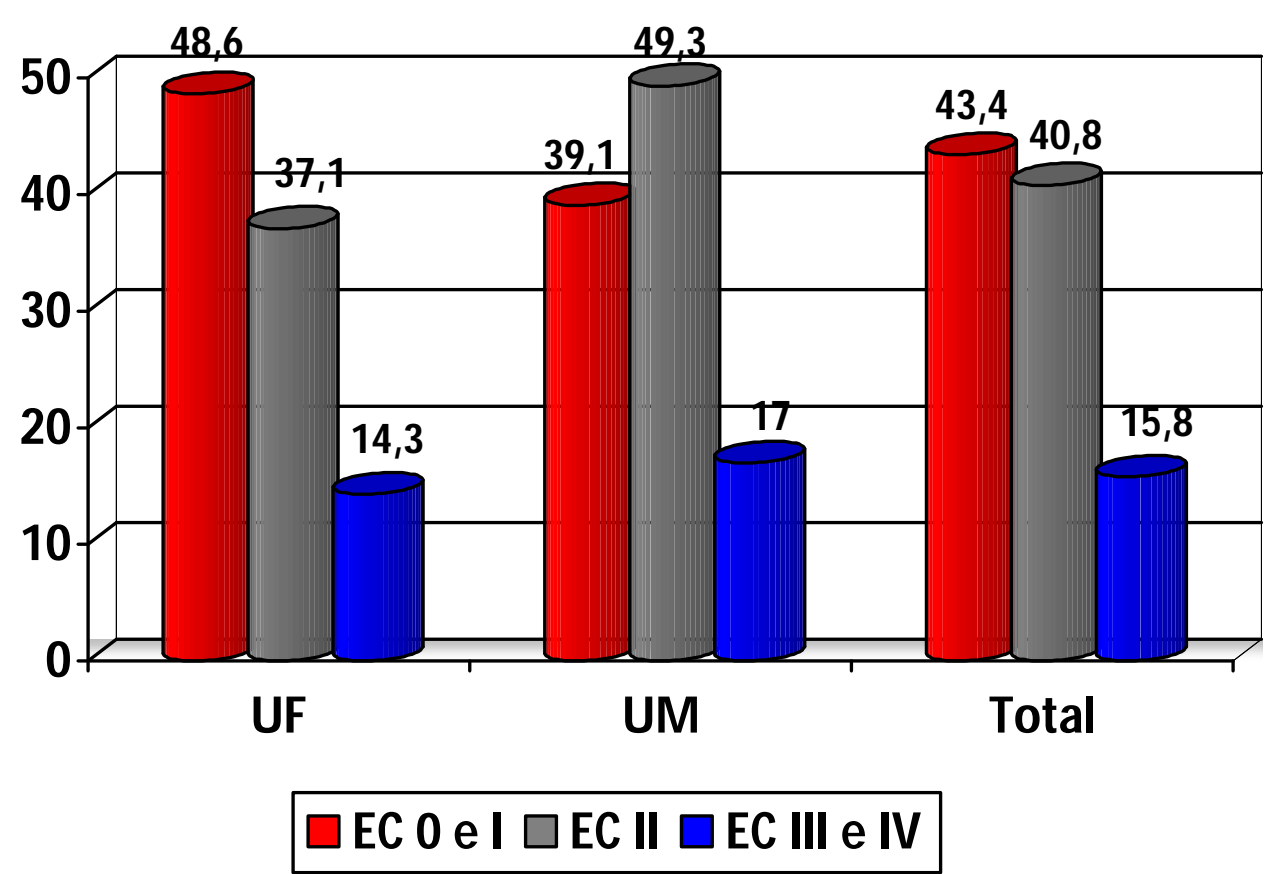

Figura 1. Perfil dos tumores detectados pelo Sistema de Rastreamento Mamográfico, onde UF = Unidade Fixa e UM = Unidade Móvel. 


\subsection{Análise do Sistema de Rastreamento}

\subsubsection{Exames}

A análise do Rastreamento foi feita com base em recomendações de reconhecimento internacional. A comparação de alguns parâmetros encontrados no presente estudo, em relação aos valores recomendados internacionalmente, estão dispostos na Tabela 8.

Tabela 8. Comparação entre os resultados do Sistema de Rastreamento com os valores recomendados por órgão internacionais de auditoria em rastreamento mamográficos.

\begin{tabular}{|c|c|c|c|c|}
\hline & EG & $\mathbf{D Q}$ & $\begin{array}{c}\text { BI- } \\
\text { RADS }\end{array}$ & SR \\
\hline Taxa de participação & $70 \%$ & $70 \%$ & & $33,1 \%$ \\
\hline Taxa de reconvocação & $<7 \%$ & $<10 \%$ & $<10 \%$ & $9,4 \%$ \\
\hline $\begin{array}{l}\text { Valor Preditivo Positivo } 1 \\
\text { Exame Alterado }\end{array}$ & & & $5-10 \%$ & $4,49 \%$ \\
\hline $\begin{array}{l}\text { Valor Preditivo Positivo } 2 \\
\text { Biópsia }\end{array}$ & & & $25-40 \%$ & $16 \%$ \\
\hline $\begin{array}{l}\text { Proporção de casos com } \\
\text { estadiamento II em relação ao } \\
\text { total diagnosticado }\end{array}$ & $<30 \%$ & & & $40,8 \%$ \\
\hline $\begin{array}{l}\text { Proporção dos casos invasivos } \\
\text { com linfonodos negativos }\end{array}$ & $>70 \%$ & $>70 \%$ & & $57,4 \%$ \\
\hline $\begin{array}{l}\text { Proporção dos casos positivos } \\
\text { com tumor menor que } 10 \mathrm{~mm}\end{array}$ & $>=25 \%$ & $>=25 \%$ & $>=30 \%$ & $27,94 \%$ \\
\hline $\begin{array}{l}\text { Taxa de detecção de câncer } \\
\text { invasivo }\end{array}$ & & $>5 / 1000$ & & $3,8 / 1000$ \\
\hline Estadiamento 0 e I & & & $>50 \%$ & $43,4 \%$ \\
\hline Linfonodo axilar positivo & & $<30 \%$ & $<25 \%$ & $42,6 \%$ \\
\hline $\begin{array}{l}\text { Número de casos positivos em } \\
1000 \text { exames realizados }\end{array}$ & & & $6-10$ & 4,23 \\
\hline
\end{tabular}

Onde: $\mathrm{EG}=$ European guidelines for quality assurance in breast cancer screening and diagnosis; $\mathrm{DQ}=$ Determinants de La qualité dês programmes organisés de déspsitage du câncer du sein; BI-RADS = Sistema de Laudos e Registro de Dados de Imagem da Mama; SR - Sistema de Rastreamento mamográfico do Hospital de Câncer de Barretos. 


\section{DISCUSSÃO}

O câncer de mama é a neoplasia mais comum entre as mulheres de todo o mundo ${ }^{1}$, com 49.400 casos esperados para 2008 no Brasil ${ }^{3}$. Essa enfermidade mostra-se como uma importante patologia que merece atenção dos gestores em saúde pública e leva a uma mobilização para o seu rastreamento ${ }^{4}$, que pode ter na utilização de UMs uma boa estratégia de rastreamento mamográfico, pois com um custo menor, podem atingir um maior número de mulheres.

A taxa de participação das mulheres no nosso programa de rastreamento foi de $33,1 \%$. Esse valor mostrou-se baixo se comparado aos sugeridos pelas recomendações internacionais, que estão em torno de 70 a $80 \%$ de cobertura no primeiro turno ${ }^{48-50}$ e também quando comparados ao ocorrido em Valência, onde a taxa de participação foi de $72,98 \%{ }^{51}$, e no Reino Unido, com $75 \%{ }^{52}$. Contudo, se confrontado com outros rastreamentos que também encontram-se em fase inicial, nossa taxa está próxima aos $48 \%$ da França ${ }^{52}, 54 \%$ da Austrália, de $11,5 \%$ a $54,7 \%$ em diferentes locais do Canadá ${ }^{48}$ e superior quando comparamos aos valores de $9,6 \%$ conseguidos pelo rastreamento realizado na região de L` Ariana na Tunísia ${ }^{53}$. Outro fator importante é a inexistência de estudos semelhantes em países em desenvolvimento, como o Brasil, o que tornariam as comparações mais próximas da realidade, exceção feita ao México onde foi realizado um estudo de rastreamento, porém os dados divulgados não são comparáveis quando observamos a taxa de participação da população. ${ }^{2}$ 
A maioria das mulheres participantes apresentou faixa etária de 40 a 49 anos (48,2\%), seguidas por mulheres de 50 a 59 anos $(34,4 \%)$ e de 60 a 69 anos $(17,4 \%)$. Isto também se deve ao fato de $45,2 \%$ da população elegível ser composta por mulheres na faixa etária mais jovem, seguidas pelas mulheres de 50 a 59 anos (31,9\%) e finalmente das mulheres de 60 a 69 anos (22,8\%), segundo dados do IBGE. Os dados deste estudo ainda não são conclusivos no que diz respeito ao início do exame mamográfico aos 40 anos. Análises com um maior números de mulheres participantes, e maior tempo de observação, poderão no futuro próximo elucidar esta questão.

O critério de seleção de mulheres na faixa etária de 40 a 69 anos foi o mesmo empregado por Seguret et al ${ }^{52}$, pelo Colégio Americano de Radiologia, pela American Cancer Society ${ }^{5}$ e por outros importantes estudos europeus, intensamente analisados ${ }^{17}$. Em estudo realizado por Shapiro foi descrito que quase metade dos programas analisados iniciou seu rastreamento em mulheres com 40 anos e em mais da metade o término se deu aos 69 anos $^{25}$. Outros trabalhos apresentam uma variação diferente na faixa etária examinada, como acontece nos relatos de Vizcaino et al ${ }^{51}$, que consideram a faixa etária de 45 a 65 anos. No presente estudo não houve significância estatística para validar a realização de exames em mulheres de 40 a 49 anos. Entretanto, a mesma série foi observada por um tempo maior onde, apesar dos dados serem preliminares, parece haver significância nesses novos dados, o que recomenda a realização do exame neste faixa etária no nosso país. Dados que estão sendo avaliados e ainda não 
publicados elucidarão melhor a grande questão sobre a realização do rastreamento mamográfico em mulheres dos 40 aos 49 anos de idade.

Mesmo que alguns estudos defendam que a periodicidade da realização dos exames de rastreamento em mulheres de 40 a 49 anos deva ser inferior a dois anos ${ }^{54}$, aqui optou-se por uma freqüência bianual, como ocorreu na maioria dos estudos representados por Shapiro ${ }^{25}$, diferentemente dos estudos ingleses que preconizam rastreamentos a cada três anos. Embora o Instituto Nacional do Câncer (INCA), instituição que representa o Ministério da Saúde, tenha lançado no ano de 2003, o consenso para rastreamento mamográfico, o qual indica que mamografia deve ser bianual em mulheres de 50 a 69 anos, não há ainda estudos nacionais sobre qual freqüência as mulheres brasileiras devam realizar exames preventivos nas diferentes faixas etárias.

Não é hábito da população brasileira se submeter ao rastreamento, entretanto observamos uma grande procura pela UM para a realização dos exames, o que vem corroborar com a premissa de que sua utilização atua como grande facilitadora ao acesso ao exame de mamografia ${ }^{55}$.

A utilização da Unidade Móvel para o rastreamento do câncer já se mostrou custo-efetiva ${ }^{12}$, além de ser uma importante estratégia para a superação de obstáculos que impedem a realização de exames pela população de poucos recursos e/ou que tenha difícil acesso ao sistema de saúde, de tal forma que as metas sejam atingidas ${ }^{55}$. Segundo dados americanos, 2,4\% das mamografias do país foram realizadas em UM, porém o número de unidades avaliadas foi baixo ${ }^{56}$. Nossos achados superam 
consideravelmente essa constatação, dado o fato de que 10.521 (58,6\%) mulheres procuraram esta unidade para a realização do seu exame, contra 7.443 que o fizeram na Unidade Fixa.

A unidade móvel realizou 10.521 exames, com uma média diária de 26,3 exames e uma taxa de ocupação de 65,7\%, número semelhante aos 31 exames realizados diariamente em um estudo em UMs nos Estados Unidos. Neste mesmo estudo observou-se que apenas 15\% das UMs apresentaram taxa de ocupação superior a apresentada no presente trabalho ${ }^{57}$. Na unidade fixa obteve-se uma taxa de ocupação da ordem de $15,5 \%$, na qual foram realizados 7.443 exames em uma média de 18,6 exames por dia.

Com base nesses dados ficou claro que as unidades não tiveram sua capacidade operacional total explorada, necessitando, portanto, de maior interação entre as cidades participantes e a administração do rastreamento, para que este desperdício seja evitado.

A convocação das mulheres para a participação do rastreamento foi realizada por vários meios. Alguns desses já possuem eficácia comprovada em países desenvolvidos, como por exemplo, o uso de carta convite, que obteve uma taxa de participação de $85 \%{ }^{58}$. Outros programas combinaram esta tática com campanhas na mídia escrita e falada, alcançando índices da ordem de $72,98 \%{ }^{51}$. Contudo, países em desenvolvimento, assim como o Brasil, ainda não contam com uma estratégia consolidada para a convocação. Provavelmente, não exista uma estratégia que seja por si só eficaz nesses países e, dessa maneira, deve-se lançar mão da aplicação de um conjunto de modalidades. 
Esse pressuposto é confirmado em nosso estudo, haja vista que as melhores estratégias de convocação foram aquelas relacionadas aos serviços de atenção primária à saúde e orientação pessoal, sendo o encaminhamento pelo PSF, principalmente através da pessoa do agente comunitário de saúde (ACS), a forma mais eficaz, com uma taxa de 46,8\% de convocações. Em seguida aparece o encaminhamento e/ou orientação médica, responsável pela convocação de $27,0 \%$ das mulheres. Este dado vem legitimar uma possível utilização do rastreamento como uma alternativa à dificuldade e demanda reprimida pelo exame de mamografia, pois devido esta situação os médicos orientariam as pacientes a procurarem 0 rastreamento para a realização de um exame de rotina.

A taxa de reconvocação do rastreamento foi de $9,4 \%$, sendo compatíveis aos níveis sugeridos internacionalmente pelas recomendações, como os do Canadá e da Europa, que estão em torno de 10\%. Estudos Europeus também apresentaram valores semelhantes ${ }^{52}$. O Breast Cancer Surveillance Consortium, US National Cancer Institute (BCSC); national breast and cancer early detection program, US Centers for Disease Control and Prevention (NBCCEDP); UK National Health Service Breast Screening Program (NHSBSP) apresentaram, respectivamente, taxas de reconvocação de $13,1 \%, 11,2 \%$ e $7,4 \%$ para o primeiro turno do rastreamento, observamos ainda uma taxa de $18,1 \%$ relatada por Bouchlaka et al. ${ }^{59} 53$

Tanto os dados de taxa de detecção de câncer quanto taxa de convocação não apresentam precedentes em território nacional, portanto difícil de avaliar se estão corretos para os padrões nacionais ou não. 
Das $474(2,63 \%)$ pacientes que foram submetidas à biópsia, 331 $(68,2 \%)$ realizaram biópsia a céu aberto, sendo as demais assistidas por core biopsy guiado por ultra-sonografia ou mamografia. Atualmente, pouco mais de $90 \%$ das pacientes é submetida a este último procedimento, menos invasivo e, conseqüentemente, com menor morbidade, o que Ihes propicia uma melhor qualidade de vida.

O valor preditivo positivo para biópsia foi baixo (16\%) quando comparado à indicação do BI-RADS que vai de 25 a $40 \%$, o que revela que um grande número de biópsia foi indicado. Este elevado número de biópsias pode estar relacionado ao fato de que os radiologistas, que apesar de experiência comprovada em mamografia, não estavam habituados aos números elevados de exames de rastreamento. Além disso, como o projeto era pioneiro optou-se por indicar um maior número de procedimentos invasivos a se correr o risco de perder o seguimento da paciente.

O programa diagnosticou 76 casos de câncer de mama nas duas unidades, sendo 41 casos na UM e 35 na UF. A taxa de detecção global foi de 4,23 casos por 1.000 exames realizados (3,9 e 4,7 casos em 1.000 exames nas unidades móveis e fixa, respectivamente) valor esse próximo ao preconizado pelo BI-RADS, que é de 6 a 10 casos/1000 mamografias ${ }^{50}$ e aos apresentados na Europa, onde estudo realizado na região de Valência, Espanha, a taxa de detecção de câncer por 1000 exames foi de $4,27^{51}$ na Holanda a taxa foi de 5, na Tunísia de 4,9 e no México de 2,1 a cada 1000 exames de mamografia. ${ }^{2,} 53,60$ Em outro estudo foram comparadas três grandes séries de rastreamento, nos quais dois foram realizados nos EUA e 
um no Reino Unido. Suas análises revelaram que a taxa de detecção variou de 8,6 a 10,1 casos por 1000 exames nesses locais ${ }^{59}$.

Um dos grandes pontos de um rastreamento é o estadiamento dos casos de câncer. Nesse estudo observa-se uma melhora importante dos resultados quando comparamos aos números nacionais. Obteve-se nos casos iniciais (EC 0 e I) uma taxa de $48,6 \%$ e $39,1 \%$ nas unidades fixa e móvel, respectivamente, o que representa uma média global do rastreamento de $43,4 \%$ desses estádios. Tais valores, quando comparados aos modestos $20 \%$ relatados no Estado de São Paulo através da Fundação Oncocentro de São Paulo (FOSP) ${ }^{61}$, e aos $14,5 \%$ observados na região de Barretos no período que antecede ao inicio do rastreamento, segundo o Registro Hospitalar de Câncer do Hospital de câncer de Barretos, mostram a eficiência e a importância do programa de rastreamento, mesmo no seu primeiro turno. Tal achado ganha ainda mais importância devido à preocupação com o baixo número de casos inicias em uma DRS relativamente pequena e que possui um Hospital de referência no tratamento de câncer.

Da mesma forma, nos casos mais avançados, também observamos melhoras promissoras dos números em comparação ao nacional e discreta distância quando comparados aos números americanos. Na unidade móvel os estádios III e IV representaram 17\% dos casos de câncer e na unidade fixa $14,3 \%$, com uma média global de 15,8\%. Esses valores ficaram muito aquém dos 40,5\% apresentados pelo RHC (antes do rastreamento) e 39\% pela FOSP. Nossos achados são também importantes em relação à 
literatura mundial, onde Thuler et al, utilizando dados nacionais de $\mathrm{RHC}$, mostraram que entre 1990 e 1994 os estádios avançados correspondiam por 52,5\% de todos os casos de câncer de mama e de 1995 a 2002 os valores foram da ordem de $45,3 \%{ }^{62}$.

Dessa maneira, comparando os dados do RHC do Hospital de Câncer de Barretos com os do programa, encontra-se uma diferença estatisticamente significativa $(p<0,001)$ entre os estadiamentos de câncer de mama que, apesar da imensa melhora na detecção de casos iniciais de desenvolvimento, ainda encontra um número muito elevado de casos avançados. Levando-se em conta que este estudo é piloto e que havia uma demanda reprimida nessa região, esses índices devem diminuir consideravelmente após ter-se sanado esta situação.

Devido à precocidade desse estudo ainda não foi possível avaliar câncer de intervalo e redução de mortalidade que em alguns estudos estão em torno de $39 \%$ das mulheres rastreadas ${ }^{17}$.

Alguns dados de auditoria que constam de recomendações internacionais também foram analisados e comparados. Pode-se informar que ainda não conseguimos nos igualar em muitos quesitos, como por exemplo, a já citada taxa de participação $(33,1 \%$ contra os $75 \%$ preconizados) e o total de casos com estadiamento II em relação ao número total de casos diagnosticados, onde nossos dados foram maiores que o esperado, possivelmente por uma falta de diagnóstico precoce. 
Os demais achados confirmam a carência de um programa de rastreamento no nosso país, o que faz com que os casos diagnosticados pelo sistema de rastreamento sejam mais avançados, como por exemplo:

- Proporção dos casos invasivos com linfonodos negativos, que deveriam ser maior que $70 \%$ e alcançamos apenas $57,4 \%$

- Estadiamento 0 e I onde os nossos números foram de 43,4\%, quando o ideal seria de $50 \%$.

- Linfonodo axilar positivo onde o preconizado é $25-20 \%$ e nossos resultados ultrapassaram $42 \%$.

Outra estatística interessante e aparentemente sem precedente no nosso território é o numero de casos de câncer invasivo por 1000 exames, onde o recomendado é de que seja maior que cinco casos, e no nosso estudo foi encontrado apenas 3,8. A princípio, por este ser um estudo pioneiro, algumas perguntas ficaram sem respostas, como o por que de nossa taxa global de detecção não ultrapassar 4,23 casos por 1000 exames, quando o recomendado pelo BI-RADS é de 6 a 10.

Parte destes dados falhos pode ser decorrente da hipótese de que as equipes técnica, radiológica, patológica e mesmos os cirurgiões, não estavam devidamente treinados para atuar em um sistema de rastreamento. Atualmente no nosso programa essas falhas foram sanadas, pois as equipes envolvidas são agora de dedicação exclusiva para o rastreamento e com isso esperamos que no futuro esses números se aproximem dos preconizados internacionalmente. 
Comparando os diagnósticos dos diferentes tipos de câncer (in situ e invasivo) em função dos aparelhos utilizados, observamos também sensível diferença a favor do mamógrafo fixo, onde a UF apresentou um melhor desempenho no diagnóstico do câncer in situ. Esta circunstância está ligada ao fato da UM estar mais propensa a alterações que levam a uma pior qualidade do exame, pois a sua característica itinerante confere-lhe tal propriedade. Apesar da realização rotineira de testes de calibragem no mamógrafo da unidade móvel, este se mostrou menos eficaz para o diagnóstico de lesões precoces. Esta deficiência pode estar relacionada desde a descalibragem do equipamento, resultado das trepidações decorrentes do deslocamento, até mesmo as condições de revelação das películas, que apesar de serem constantemente monitoradas sofrem as mesmas intempéries de estar em uma unidade em movimento.

Embora tenhamos obtido dados concisos e sucesso na implantação do sistema de rastreamento mamográfico o grande viés metodológico do estudo deve-se ao fato da não separação das mulheres sintomáticas das assintomáticas. Tal iniciativa foi tomada segundo a filosofia da Instituição, na qual o Departamento de Prevenção está incluído, e também devido à demanda reprimida de mamografia na região, fato que se confirma pelo elevado número de encaminhamentos médicos para o sistema de rastreamento. Conseqüentemente, o déficit maior é dado pela falta de uma política de saúde prioritária para rastreamento mamográfico.

O armazenamento dos dados do rastreamento apareceu como outro problema técnico, pois o cadastro das pacientes era inserido no banco de 
dados geral da instituição. O ideal seria que os prontuários das pacientes que fazem parte do rastreamento fossem arquivados em locais específicos no Serviço de Arquivo Médico e Estatística (SAME), facilitando assim seu manuseio sob qualquer aspecto. Atualmente, os exames normais são entregues às pacientes junto a uma orientação de levá-los consigo no próximo exame de rastreamento, mas o que observamos hoje é o esquecimento, na maioria das vezes, deste laudo. A realidade atual da Instituição impede o armazenamento das películas em suas dependências, dado o fato de ainda não possuir espaço físico suficiente para tal procedimento. No entanto, tem-se na digitalização dos exames a solução desse problema, tecnologia essa que hoje esbarra em seu custo elevado, mas que num futuro não muito distante poder-se-á tornar rotina.

Portanto o programa de rastreamento foi implantado, se encontra em operação e seus dados até o momento se mostram aceitáveis, porém devem ser aprimorados. De uma maneira geral observa-se que ainda tem muito a progredir e como este é um estudo piloto, sem precedentes em números e achados obtidos em nível nacional, todos os resultados apresentaram-se satisfatórios e não ideais.

Acredita-se que após alguns anos e novas análises em nossos dados, poder-se-á traçar um perfil do rastreamento brasileiro propriamente dito, mesmo porque nada foi encontrado na literatura nacional, no tocante ao rastreamento mamográfico realizado na prática. Somente dessa forma teremos mais informação com embasamento prático-científico e poderemos então, ter subsídios para indicar a idade, periodicidade e demais índices aos 
quais se poderá seguir e compilá-los apresentando assim um parâmetro nacional. 
CONCLUSÃO 


\section{CONCLUSÃO}

Tendo em vista a dificuldade da implementação de um sistema inédito de rastreamento mamográfico num país em desenvolvimento e a necessidade de se oferecer um serviço de qualidade para a população com dificuldades no acesso ao sistema público de saúde, concluímos que esse promissor programa de rastreamento é viável no território nacional e que apesar da precocidade dos resultados, estes são satisfatórios. Dessa maneira o programa deve dar continuidade as suas atividades, pois estaremos aptos a diagnosticar cada vez mais casos precoces de câncer de mama e assim inferir redução nas taxas de mortalidade decorrente dessa doença. 
REFERÊNCIAS BIBLIOGRÁFICAS 


\section{REFERÊNCIAS BIBLIOGRÁFICAS}

1. Parkin DM, Fernandez LM. Use of statistics to assess the global burden of breast cancer. Breast J;12 Suppl 1:S70-80. 2006.

2. Rodriguez-Cuevas S, Guisa-Hohenstein F, Labastida-Almendaro S. First breast cancer mammography screening program in Mexico: initial results 2005-2006. Breast J;15(6):623-31. 2009.

3. INCA INdC-. Estimativas 2008: Incidência de Câncer no Brasil. Brasil Ministério da Saúde Secretaria de Atenção à Saúde Instituto Nacional de Câncer Coordenação de Prevenção e Vigilância de Câncer; 2007.

4. Humphrey LL, Helfand M, Chan BK, Woolf SH. Breast cancer screening: a summary of the evidence for the U.S. Preventive Services Task Force. Ann Intern Med;137(5 Part 1):347-60. 2002.

5. Smith RA, Saslow D, Sawyer KA, Burke W, Costanza ME, Evans WP, 3rd, et al. American Cancer Society guidelines for breast cancer screening: update 2003. CA Cancer J Clin;53(3):141-69. 2003.

6. Miller AB, To T, Baines CJ, Wall C. The Canadian National Breast Screening Study-1: breast cancer mortality after 11 to 16 years of follow-up. A randomized screening trial of mammography in women age 40 to 49 years. Ann Intern Med;137(5 Part 1):305-12. 2002.

7. Committee on technologies for the early detection of breast cancer. Mammography and Beyond: Developing Technologies for the early detection of breast cancer. Washington, DC: National Academy Press; 2001.

8. Moody-Ayers SY, Wells CK, Feinstein AR. "Benign" tumors and "early detection" in mammography-screened patients of a natural cohort with breast cancer. Arch Intern Med;160(8):1109-15. 2000. 
9. Wilson JM, Jungner YG. [Principles and practice of mass screening for disease]. Bol Oficina Sanit Panam;65(4):281-393. 1968.

10. Lee EE, Thomas CA, Vu T. Mobile and portable dentistry: alternative treatment services for the elderly. Spec Care Dentist;21(4):153-5. 2001.

11. Steinberg ME. A mobile mammography program in the workplace. Successful program. AAOHN J;49(7):325-8. 2001.

12. Mauad EC. Efetividade da Unidade Móvel para rastreamento de câncer de mama e de colo uterino [Doutorado]. São Paulo: Universidade Federal de São Paulo; 2007.

13. Peek ME, Han J. Mobile mammography: assessment of self-referral in reaching medically underserved women. J Natl Med Assoc;99(4):398-403. 2007.

14. Parkin DM, Bray F, Ferlay J, Pisani P. Global cancer statistics, 2002. CA Cancer J Clin;55(2):74-108. 2005.

15. Ascunce EN, Moreno-Iribas C, Barcos Urtiaga A, Ardanaz E, Ederra Sanz M, Castilla J, et al. Changes in breast cancer mortality in Navarre (Spain) after introduction of a screening programme. J Med Screen;14(1):1420. 2007.

16. Tabar L, Vitak B, Chen HH, Duffy SW, Yen MF, Chiang CF, et al. The Swedish Two-County Trial twenty years later. Updated mortality results and new insights from long-term follow-up. Radiol Clin North Am;38(4):625-51. 2000 .

17. Duffy SW, Tabar L, Chen HH, Holmqvist M, Yen MF, Abdsalah S, et al. The impact of organized mammography service screening on breast carcinoma mortality in seven Swedish counties. Cancer;95(3):458-69. 2002. 
18. Nystrom L, Rutqvist LE, Wall S, Lindgren A, Lindqvist M, Ryden S, et al. Breast cancer screening with mammography: overview of Swedish randomised trials. Lancet;341(8851):973-8. 1993.

19. Parkin DM. Global cancer statistics in the year 2000. Lancet Oncol;2(9):533-43. 2001.

20. Anderson BO, Shyyan R, Eniu A, Smith RA, Yip CH, Bese NS, et al. Breast cancer in limited-resource countries: an overview of the Breast Health Global Initiative 2005 guidelines. Breast J;12 Suppl 1:S3-15. 2006.

21. Shapiro S. Evidence on screening for breast cancer from a randomized trial. Cancer;39(6 Suppl):2772-82. 1977.

22. Shapiro S. Periodic screening for breast cancer: the HIP Randomized Controlled Trial. Health Insurance Plan. J Natl Cancer Inst Monogr(22):27-30. 1997.

23. Tabar L, Chen HH, Fagerberg G, Duffy SW, Smith TC. Recent results from the Swedish Two-County Trial: the effects of age, histologic type, and mode of detection on the efficacy of breast cancer screening. J Natl Cancer Inst Monogr(22):43-7. 1997.

24. Ringash J. Preventive health care, 2001 update: screening mammography among women aged 40-49 years at average risk of breast cancer. CMAJ;164(4):469-76. 2001.

25. Shapiro S, Coleman EA, Broeders $M$, Codd $M$, de Koning $H$, Fracheboud $\mathrm{J}$, et al. Breast cancer screening programmes in 22 countries: current policies, administration and guidelines. International Breast Cancer Screening Network (IBSN) and the European Network of Pilot Projects for Breast Cancer Screening. Int J Epidemiol;27(5):735-42. 1998.

26. Tabar L, Fagerberg G, Duffy SW, Day NE, Gad A, Grontoft O. Update of the Swedish two-county program of mammographic screening for breast cancer. Radiol Clin North Am;30(1):187-210. 1992. 
27. Blanks RG, Moss SM, McGahan CE, Quinn MJ, Babb PJ. Effect of NHS breast screening programme on mortality from breast cancer in England and Wales, 1990-8: comparison of observed with predicted mortality. BMJ;321(7262):665-9. 2000.

28. Zotov V, Shyyan R. Introduction of breast cancer screening in Chernihiv Oblast in the Ukraine: report of a PATH Breast Cancer Assistance Program experience. Breast J;9 Suppl 2:S75-80. 2003.

29. Robles SC, Galanis E. Breast cancer in Latin America and the Caribbean. Rev Panam Salud Publica;11(3):178-85. 2002.

30. Modeste NN, Caleb-Drayton VL, Montgomery S. Barriers to early detection of breast cancer among women in a Caribbean population. Rev Panam Salud Publica;5(3):152-6. 1999.

31. Sasieni P. Evaluation of the UK breast screening programmes. Ann Oncol;14(8):1206-8. 2003.

32. Frelix GD, Rosenblatt R, Solomon M, Vikram B. Breast cancer screening in underserved women in the Bronx. J Natl Med Assoc;91(4):195200. 1999.

33. Alexy BB, Elnitsky C. Rural mobile health unit: outcomes. Public Health Nurs;15(1):3-11. 1998.

34. Levin JR, Hirsch SH, Bastani R, Ganz PA, Lovett ML, Reuben DB. Acceptability of mobile mammography among community-dwelling older women. J Am Geriatr Soc;45(11):1365-70. 1997.

35. Reding DJ, Lappe KA, Krueger M, Kolehouse BL, Steneil D, Leer RA. Cancer screening and prevention in rural Wisconsin: the Greater Marshfield Experience. Wis Med J;96(8):32-7. 1997. 
36. Njor SH, Olsen AH, Bellstrom T, Dyreborg U, Bak M, Axelsson C, et al. Mammography screening in the county of Fyn. November 1993-December 1999. APMIS Suppl(110):1-33. 2003.

37. Gerard K, Brown J, Johnston K. UK breast screening programme: how does it reflect the Forrest recommendations? J Med Screen;4(1):10-5. 1997.

38. Baxter N. Preventive health care, 2001 update: should women be routinely taught breast self-examination to screen for breast cancer? CMAJ;164(13):1837-46. 2001.

39. Baines CJ. The Canadian National Breast Screening Study: a perspective on criticisms. Ann Intern Med;120(4):326-34. 1994.

40. Alexander FE, Anderson TJ, Brown HK, Forrest AP, Hepburn W, Kirkpatrick $A E$, et al. 14 years of follow-up from the Edinburgh randomised trial of breast-cancer screening. Lancet;353(9168):1903-8. 1999.

41. Teh W, Wilson AR. The role of ultrasound in breast cancer screening. A consensus statement by the European Group for Breast Cancer Screening. Eur J Cancer;34(4):449-50. 1998.

42. Saslow D, Boetes C, Burke W, Harms S, Leach MO, Lehman CD, et al. American Cancer Society guidelines for breast screening with $\mathrm{MRI}$ as an adjunct to mammography. CA Cancer J Clin;57(2):75-89. 2007.

43. Lewin JM, D'Orsi CJ, Hendrick RE, Moss LJ, Isaacs PK, Karellas A, et al. Clinical comparison of full-field digital mammography and screen-film mammography for detection of breast cancer. AJR Am $J$ Roentgenol;179(3):671-7. 2002.

44. Skaane P. Studies comparing screen-film mammography and full-field digital mammography in breast cancer screening: updated review. Acta Radiol;50(1):3-14. 2009. 
45. Rosenberg RD, Hunt WC, Williamson MR, Gilliland FD, Wiest PW, Kelsey CA, et al. Effects of age, breast density, ethnicity, and estrogen replacement therapy on screening mammographic sensitivity and cancer stage at diagnosis: review of 183,134 screening mammograms in Albuquerque, New Mexico. Radiology;209(2):511-8. 1998.

46. Nystrom L, Andersson I, Bjurstam N, Frisell J, Nordenskjold B, Rutqvist LE. Long-term effects of mammography screening: updated overview of the Swedish randomised trials. Lancet;359(9310):909-19. 2002.

47. Pivot X, Rixe O, Morere J, Coscas Y, Cals L, Namer M, et al. Breast cancer screening in France: results of the EDIFICE survey. Int $\mathrm{J}$ Med Sci;5(3):106-12. 2008.

48. Canada H. Quality determinants of organized breast cancer screening programs in Canada. Ottawa (ON): Minister of Public Works and Government Services Canada; 2003.

49. European guidelines for quality assurance in breast cancer screening and diagnosis. 4 ed. Luxembourg: European Communities; 2006.

50. American College of Radiology (ACR). ACR BI-RADS Mammography. ACR breast imaging reporting and data system, breast imaging atlas. 4 ed. Reston, VA: American College of Radiology; 2003.

51. Vizcaino I, Salas D, Vilar JS, Ruiz-Perales F, Herranz C, Ibanez J. Breast cancer screening: first round in the population-based program in Valencia, Spain. Collaborative Group of Readers of the Breast Cancer Screening Program of the Valencia Community. Radiology;206(1):253-60. 1998.

52. Seguret F, Daures JP, Guizard AV, Mathieu-Daude H, Bonifacj JC, Cherifcheik J, et al. Herault breast screening programme: results after 30 months of a mobile French schedule. Eur J Cancer Prev;4(4):299-305. 1995. 
53. Bouchlaka A, Ben Abdallah M, Ben Aissa R, Zaanouni E, Kribi L, Smida $S$, et al. [Results and evaluation of 3 years of a large scale mammography program in the Ariana area of Tunisia]. Tunis Med;87(7):43842. 2009.

54. Bjurstam N, Bjorneld L, Duffy SW, Smith TC, Cahlin E, Erikson O, et al. The Gothenburg Breast Cancer Screening Trial: preliminary results on breast cancer mortality for women aged 39-49. J Natl Cancer Inst Monogr(22):53-5. 1997.

55. Dershaw DD, Liberman L, Lippin BS. Mobile mammographic screening of self-referred women: results of 22,540 screenings. Radiology;184(2):415-9. 1992.

56. Brown ML, Fintor L. U.S. screening mammography services with mobile units: results from the National Survey of Mammography Facilities. Radiology;195(2):529-32. 1995.

57. DeBruhl ND, Bassett LW, Jessop NW, Mason AM. Mobile mammography: results of a national survey. Radiology;201(2):433-7. 1996.

58. Ascunce N, del Moral A, Murillo A, Alfaro C, Apesteguia L, Ros J, et al. Early detection programme for breast cancer in Navarra, Spain. Eur J Cancer Prev;3 Suppl 1:41-8. 1994.

59. Smith-Bindman R, Chu PW, Miglioretti DL, Sickles EA, Blanks R, Ballard-Barbash $\mathrm{R}$, et al. Comparison of screening mammography in the United States and the United kingdom. JAMA;290(16):2129-37. 2003.

60. Duijm LE, Groenewoud JH, Jansen FH, Fracheboud J, van Beek M, de Koning HJ. Mammography screening in the Netherlands: delay in the diagnosis of breast cancer after breast cancer screening. $\mathrm{Br} J$ Cancer;91(10):1795-9. 2004. 
61. Fundação Oncocentro de São Paulo F. Boletim do registro Hospitalar de Câncer. 2008 [updated 2008; cited]; Available from: http://www.fosp.saude.sp.gov.br/docs/boletins/rhc18.pdf.

62. Thuler LCS. Estadiamento inicial dos casos de câncer de mama e colo do útero em

mulheres brasileiras. Revista Brasileira de Ginecologia e Obstetricia 27:656-60. 2005. 
ANEXOS 


\section{MAMOGRAFIA Ficha de Cadastro}

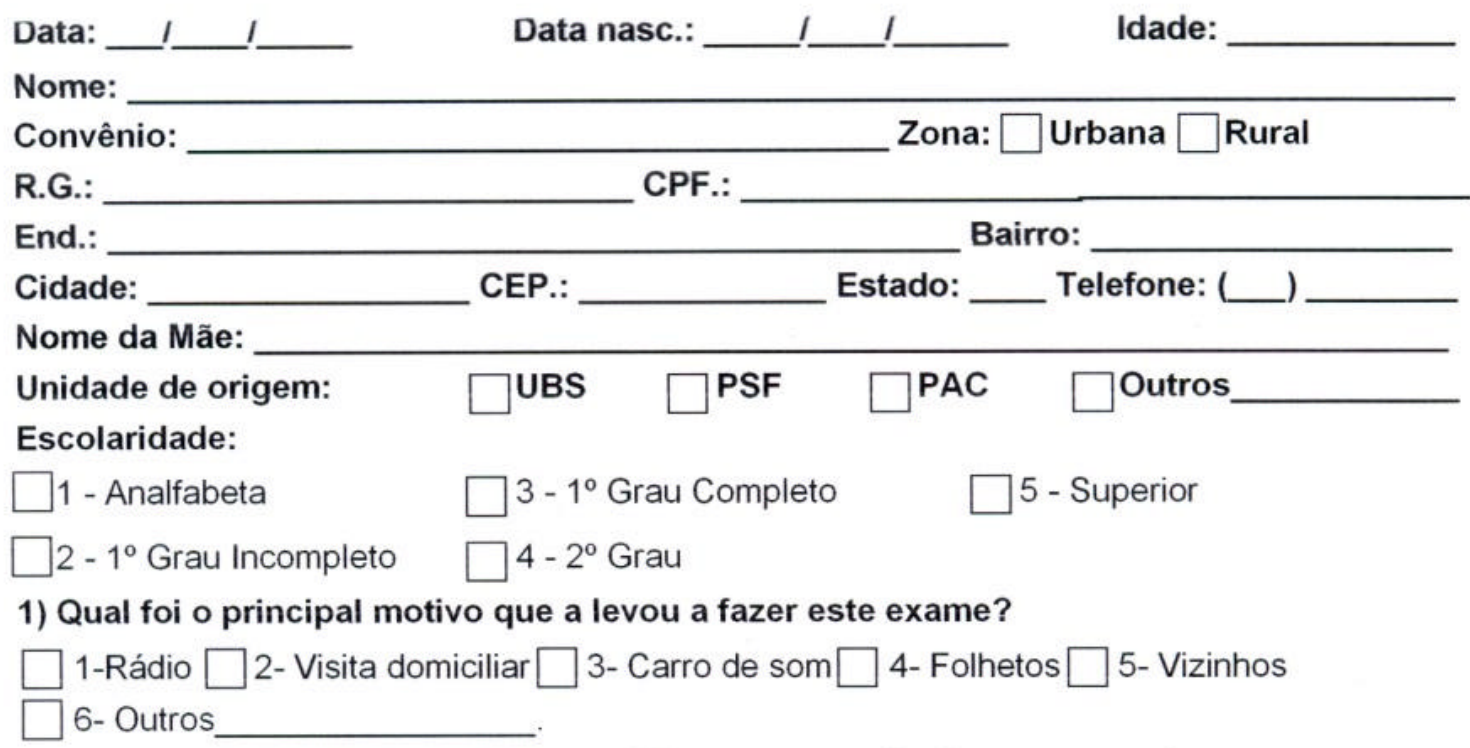

2) Mamografia é uma chapa da mama feito por uma máquina que pressiona a mama. Você já fez Mamografia alguma vez?

$\square 1$ - Sim $\quad \square 2$ - Não $\quad \square 9$ - Ignorado (Não sei)

3) Há quanto tempo

4) Local da realização deste exame.

$\square$ Hospital Pio XII

$\square$ Ônibus

5) Notou alterações na mama.

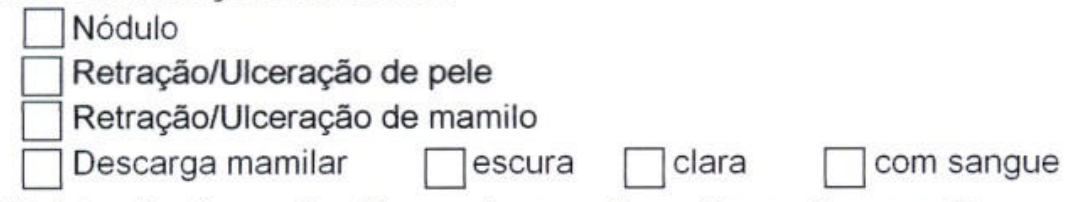

6) Existe alguém na familia que teve ou tem câncer de mama?

7) Classe Sócio-Econômico (anotar a quantidade na casa)

$\begin{array}{lll}\text { ( ) Carro } & (\text { ) TV em Cores } & (\text { ) Video Cassete } \\ \text { ( ) Rádio } & (\text { ) Banheiro } \\ \text { ( ) Empregada Mensalista } & (\text { ) Aspirador de pó } \\ \text { ( ) Máquina de Lavar Roupa } & (\text { ) Geladeira }\end{array}$


ANEXO II
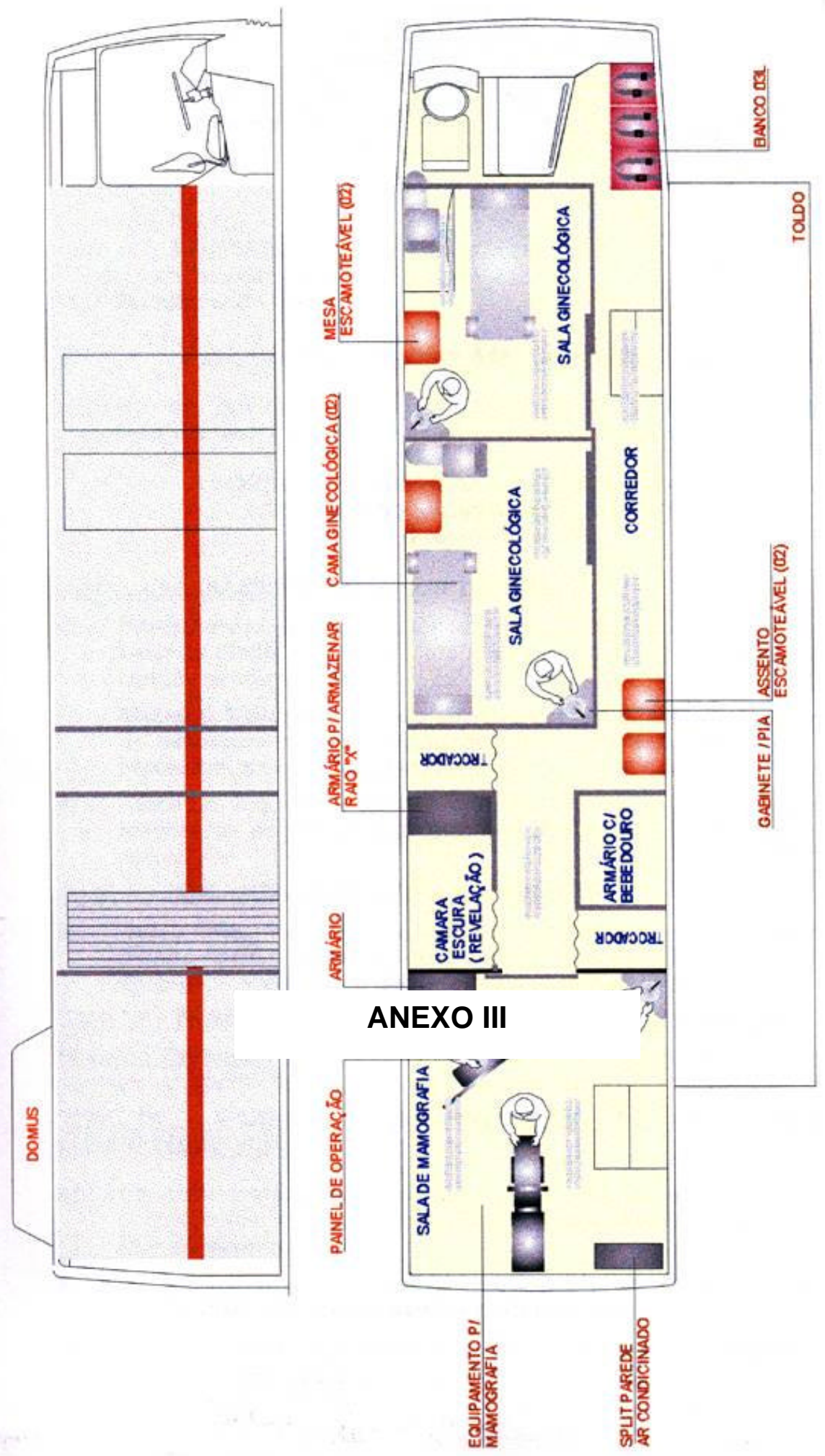
ANEXO III
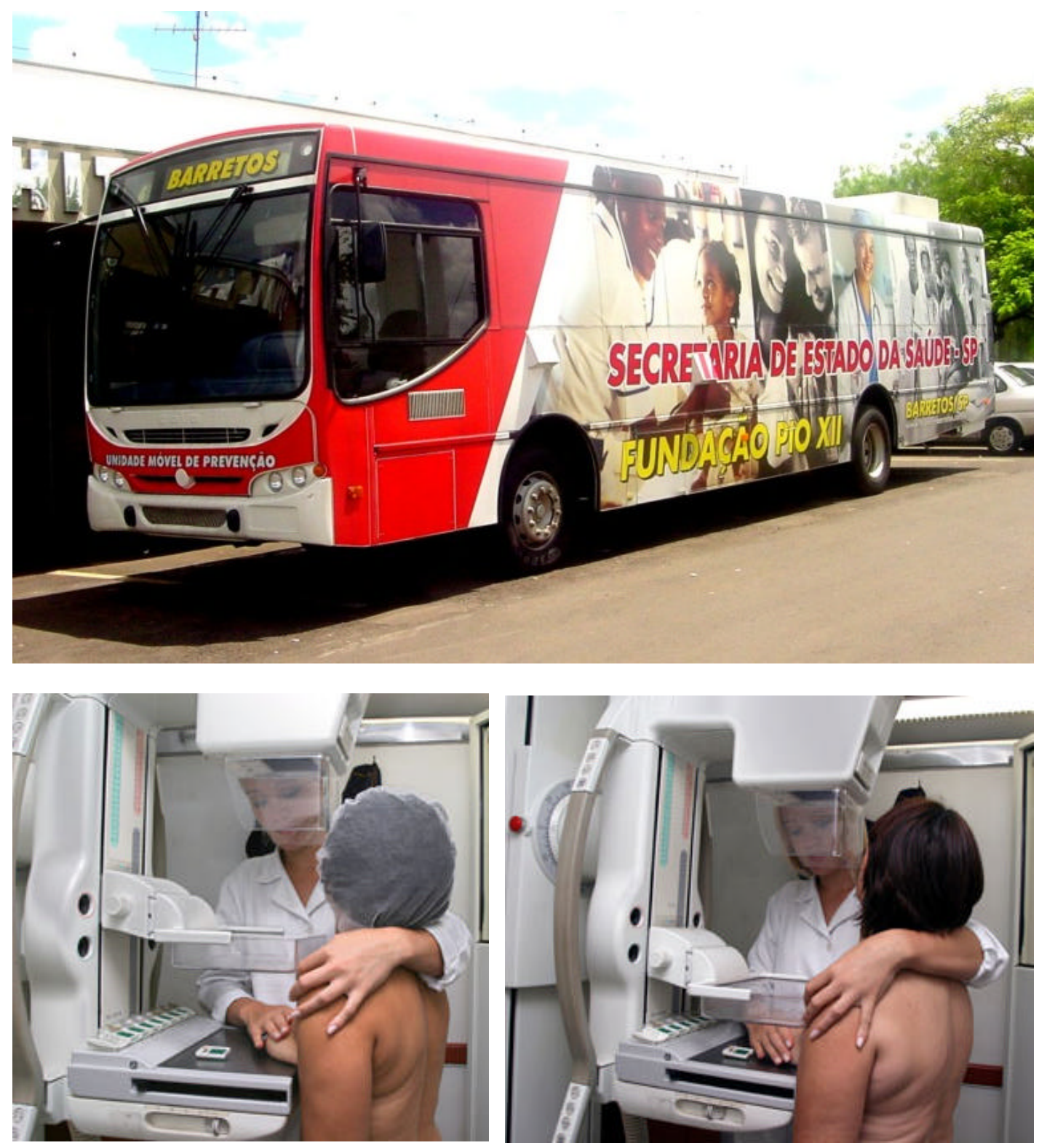


\section{ANEXO IV}

\section{Fundaçăo}

PioXII

\section{HOSPITAL DO CÂNCER DE BARRETOS \\ Prevenção do Câncer de Mama}

Nome:

$\mathrm{N}^{\circ}$ prontuário:

Data da consulta:

Idade:

Sexo:

Grupo: (0) mamografia (1) exame físico

Instrução: (0) nula

(3) $2 \underline{\text { o grau incompleto }}$

(4) $3 \underline{o}$ grau completo:

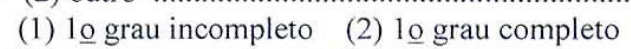

(4) $2 \underline{\text { o }}$ grau completo (5) $3 \underline{0}$ grau incompleto

(9) ignorado

Procedência:

(01) Altair (02)Barretos (03)Bebedouro (04)Cajobi (05)Colina (06)Colômbia

(07)Embaúba (08)Guaíra (09)Guaraci (10)Jaborandi (11)Monte Azul Paulista

(12)Olímpia (13)Pirangi (14)Severina (15)Taiaçu (16)Taiúva

(17)Terra Roxa (18)Viradouro (19)Vista Alegre do Alto (20)outro:

História:

Mastodinia:

(0) Não

(1) Sim perimenstrual

(0) Não

Nodulação Mamária:

Sintoma outro:

(0) Não

(1) $\operatorname{Sim}$.

(2) Sim outro

(1) Sim: ....

\section{Sintoma outro:}

Mamografia pregressa:

(0) Não

(1) $\operatorname{Sim}$ (número)

Tempo de mamografia anterior (anos): (0) 0 (1) 1 (2) 2 (3) 3 (4) $>3$

Achado mamografia anterior:

Ultrasonografia pregressa:(0) Não (1) Sim (número) Achado ultrasonografia anterior:

Gestações: $\mathrm{G} \_\mathrm{C} \_\mathrm{P}$

Menarca (anos):

Idade primeira gestação (anos):

Amamentação

(0) Não

(99) ignorado

(99) ignorado

Hormônios:

Anticoncepcional $(0)$ Não

Reposição Hormonal

(1) $\mathrm{Sim}$ (período)

(1) $\mathrm{Sim}$ (período):

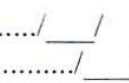




\section{$\sqrt{\text { Fundeaso }}$ iN}

Data da Mamografia:

Laudo da Mamografia (BI-RADS):

(0) 0

(1) I

(2) II

(3) III

(4) IV

(5) $\mathrm{V}$

Achado Mamográfico:
(1) BI-RADS IV e V
(3) Assimetria mamária:
(4) Calcificação:
(5) Microcalcificação:
(6) Imagem nodular:
(7) Alteração cutânea:
(8) Retração do parênquima
(9) Outro:

(0) BI-RADS I e II

Exame Físico: (0) Normal (1)Alterado Mama (2)Alt. Axila Cirurgia Pregressa: (0) ausente (1) presente:

Simetria:

(0) normal

(2) Mamas densas

(3) Alt. Fossa
Descarga papilar: (0) ausente (1) presente:

Mama:

Fossa supraclavicular:

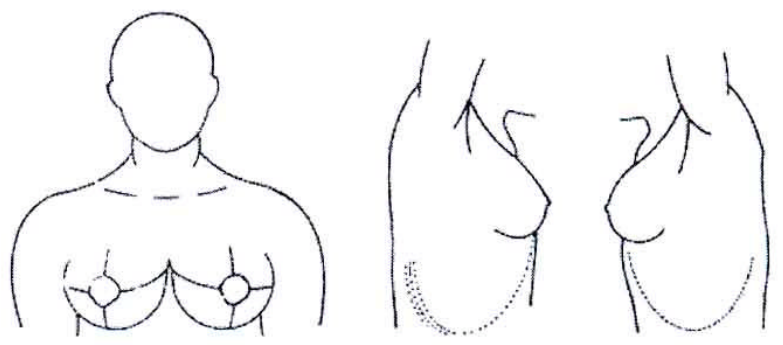

Hipótese Diagnóstica:

Exame Solicitado:

(0) Nenhum

(1) Magnificação Mamária

(2) Ultrasonografia Mamária

(3) Biópsia cirúrgica ambulatorial

(4) PAAF

(5) Outro:

(6) Ressecção cirúrgica/ Pré-operatório

Conduta:

Retorno: Médico: 


\section{APÊNDICE}

\section{Mammography-based screening program for early detection of breast cancer}

using a mobile unit: preliminary results of first round in a Brazilian rural area.

Raphael Luiz Haikel Junior ${ }^{1}$, MD, M BA; Edmundo Carvalho M auad ${ }^{1}$, MD, MBA, PhD; Luciano Fernandes Chala ${ }^{2}, M D$, PhD; Thiago Buosi Silva ${ }^{1}, M^{2}$ Sc; Adhemar LongattoFilho ${ }^{3,4}$, M Sc, PhD, PM IAC; Nestor de Barros, PhD ${ }^{5}$.

${ }^{1}$ Barretos Cancer Hospital - Departments of Cancer Prevention, São Paulo, Brazil ${ }^{2}$ Oswaldo Cruz German Hospital ${ }^{3}$ Department of Pathology of M edical School of São Paulo University (LIM 14) ${ }^{4}$ Life and Health Sciences Research Institute (ICVS), School of Health Sciences, University of M inho, Braga, Portugal

${ }^{5}$ Radiology Institute of Medical School of São Paulo University

Type of article: original research

Address for correspondence:

Edmundo C. M auad, M D, PhD

Barretos Cancer Hospital

Rua Antenor Duarte Villella, 1331

Bairro Dr. Paulo Prata

14784-400, Barretos, SP, Brazil

Tel./Fax: +55 - 17 - 3321-6600 or -6789

e-mail: prevencao@hcancerbarretos.com.br 


\section{ABSTRACT}

Introduction: Breast cancer is the most prevalent malignancy among women worldwide and accounts for $23 \%$ of all female cancers. The purpose of this study was to evaluate the effects of implementing a mammography-based, breast cancer screening program for women who live in the Barretos county area using a mobile unit (MU) and a fixed unit (FU). Methods: A total of 54,238 women aged 40 to 69 years live in this area and were eligible for breast cancer screening. Epidemiologicbased data supported the study design, and the women were examined from April 01, 2003 to March 31, 2005. Statistical analysis was used to evaluate clinical parameter frequencies and tumor characteristics using the chi-square test and Bonferroni correction test, with a level of significance of $p<0.05$. Results: Overall, 17,964 women (median age: 50 years) were effectively examined by mammography, which represented $33.1 \%$ of all eligible women. Averages of 18.6 and 26.3 women were examined per day in a FU and MU, respectively. Seventy-six patients were diagnosed with breast cancer ( 41 , or $54 \%$, in the $M U$ ), which represents 4.2 cases of breast cancer per 1,000 examinations. A significant difference in the number of cancers detected was observed between women aged 50 to 59 years and 60 to 69 years $(p \varangle 0.001)$ and between women aged 40 to 49 years and 60 to 69 years $(p \varangle 0.001$ ). No such difference was observed between women aged 40 to 49 years and 50 to 59 years $(p=0.164)$. Conclusions: This program for mammogram screening is feasible for implementation in a Brazilian territory, and the preliminary results are encouraging.

Key words: breast cancer, cancer screening, cancer prevention, mammogram 


\section{Introduction}

Breast cancer is the most frequent malignancy among women worldwide and accounts for $23 \%$ of all female cancers. It is the second leading cause of death among this population. It is estimated that there will be 192,370 cases of breast cancer in 2009 and 40,170 women will die of this disease [1]. According to the Brazilian National Institute of Cancer (INCA), 49,240 new cases are expected in the year 2010.

The main goal of cancer screening is to detect incipient tumor in order to enhance treatment efficacy and contribute to a positive outcome. Mammography is the first option for breast cancer screening, and mobile units (MUs) are believed to increase the number of women examined in screening programs, especially women in remote areas who can be provided with assistance. North America, Australia, and some European countries are the most affected by high annual indexes of new cases. The global survival rate in developed countries is $73 \%$ and in developing countries, $57 \%$. Organized screening of the general population can enhance the survival rates in $81 \%$ in 5 years [3].

The Brazilian Ministry of Health encourages breast self-examination and refers patients for mammography only for diagnostic purposes. Guidelines for breast cancer screening were introduced in 2003 just as a recommendation, but they were not part of a governmental screening program. There has been convincing evidence that organized screening effectively reduces breast cancer mortality, and this has encouraged us to initiate a screening program in rural areas of Barretos and neighboring areas [4-6]. 
The aim of this study was to evaluate the preliminary results of an organized program for detecting breast cancer introduced in rural areas of São Paulo State and to evaluate the possible impact of this strategy on Public Health cancer control. 


\section{MATERIALS AND METHODS}

The program was implemented in the Barretos countryside and in another 19 adjacent municipalities. The results herein reported are from women examined from April 2003 to March 2005 (inclusive). The population targeted was women aged 40 to 69 years, and this corresponded to 54,238 eligible women living in rural and urban area.

Exclusion criteria consisted of women who had undergone mammograms for up to 2 years and those with a previous history of breast cancer. For statistical analysis, the result of the first mammogram was considered for evaluation.

The program was initially disclosed among communitary agents and social assistants through regular meetings for clarification of goals and other steps of the program.

The screening program was authorized by the IRB of this institution, and it is registered as number 029/2006 in the institution.

\section{Screening Units}

We used 2 screening units to perform the mammograms: one Mobile Unit (MU) and another Fixed Unit (FU) located in the Department of Imaginology at the Hospital of Cancer in Barretos. The MU has one set of equipment for mammography and the FU has 3 sets, providing a capacity for 40 examinations in the MU and 120 examinations in the FU. 


\section{Classification report}

The BI-RADS classification system was used to report the findings observed in the mammograms. Women with mammograms classified as BI-RADS 1 and BIRADS 2 were classified as normal and no further action was taken. Classification BIRADS 3 was not considered, in screening exam. Women with mammograms classified as BI-RADS 4 and BI-RADS 5 were referred for further examinations. Women with carcinoma were treated in Barretos Cancer Hospital; women with no neoplastic lesions were referred to the Hospital according to its guidelines.

\section{Quality assurance}

Quality assurance and quality control followed the "European guidelines for quality assurance in breast cancer screening and diagnosis," the "Determinants de la qualité dês programmes organisés des déspistage du câncer du sein, Canada 2003," and the Brazilian edition of the BI-RADS system.

\section{Statistical analysis}

Statistical analysis was performed with the chi-square test and Bonferroni correction test, with a level of significance of $p \varangle 0.05$. Clinical and pathological data were stored and analyzed using the SPSS statistical software (version 16.0, SPSS Inc., Chicago, IL, USA). 


\section{RESULTS}

A total of 17,964 mammograms were performed, which means that $33.1 \%$ of all eligible women participated in the screening. In the MU, 10,521 (58.6\%) examinations were performed. Averages of 18.6 and 26.3 women were examined per day in the FU and MU, respectively. The average age of these women was 51 years, and the median age was 50 years. The predominant age range was 40-49 years (48.2\%), followed by 50-59 years (34.4\%) and 60-69 years (17.4\%).

Of the women, $42.1 \%(n=7,560)$ declared that they had never had a mammogram before, and $57.9 \%(10,404)$ stated that they had undergone at least one mammogram before.

The strategies to motivate adherence to the program showed that use of communitary agents is the most efficient way to persuade women to adhere to the screening program $(46.8 \%$ of women declared that they were convinced by communitary agents). Other sources contributing to adherence to the program consisted of physician influence $(27.1 \%)$, radio $(12.4 \%)$, conversations with friends (5.7\%), and others (8.1\%).

A complementary examination was necessary for $9.4 \%(1,690 / 17,964)$ of the women, and biopsies were taken from $2.63 \%(474 / 17,964)$ of the women. Seventysix cases of breast cancer were identified: $41(53.9 \%)$ from examination in the MU and $35(46.1 \%)$ from examination in the FU. The positive predictive value (PPV1), which determines how many abnormal examinations are consistent with cancer, was $4.49 \%(76 / 1,690)$. The positive predictive value (VPP2), which determines the precise indication for the biopsies, was $16 \%(76 / 474)$. 
The most frequent histological types of breast cancer were invasive ductal carcinoma (72.4\%), invasive lobular carcinoma (17.1\%), and ductal carcinoma in situ (10.5\%)(Table 1). Mammograms performed in the FU detected significantly more cases of in situ carcinoma than mammograms performed in the MU $(p \varangle 0.001)$.

Considering the number of mammograms performed, we identified 4.2 cases of breast cancer per 1,000 examinations. Cancer occurred more frequently in the 60-69 year-old group, with 6.7 cases / 1,000 examinations, followed by the 40 49 year-old group with 3.9 cases/ 1,000 examinations, and the 50-59 year-old group with 3.4 cases/1,000 examinations. Cancer detection was more frequent in patients examined in the FU (4.7 cases/1,000 examinations) than in the MU (3.9 cases/ 1,000 examinations) $(p \triangleleft 0.001)$.

The numbers of cancers detected were significantly different between women aged 50-59 years and 60-69 years $(p \varangle 0.001)$ and between women aged 4049 years and $60-69$ years ( $p \varangle 0.001$ ), but not between women aged $40-49$ years and $50-59$ years $(p=0.164)$.

The clinical stages of the tumors, according to the Classification of M alignant Tumors (TNM) of the International Union Against Cancer (UICC), showed that of the total, $43.4 \%$ of the tumors were in the early stages of development (EC 0 and EC I). It was also found that $48.6 \%$ of tumors detected in the FU and $39.1 \%$ of tumors detected in the MU were in the same stage. Tumors in advanced stages (CS III and CS IV) accounted for $15.8 \%$ of cases, and $14.3 \%$ were detected in the FU and $17 \%$ in the MU. Assuming that there is no difference in staging between the Fixed and M obile Units, the probability of this difference being explained by chance is $0.3 \%$. 
Therefore, it was concluded that there is a statistically significant difference in staging between the units $(p=0.003)$. 


\section{DISCUSSION}

Breast cancer is a major form of pathology that requires the attention of managers in public health and leads to a call for screening and early detection aimed at reducing the associated morbidity and mortality at the population level [26]. Given the difficulty of implementing a unique system of mammographic screening in a developing country and the need to offer a quality service to people with difficulties in accessing the public health system, it was agreed that: 1) the screening program is feasible to implement in a national territory, 2) reproduction of the screening program is feasible, and 3) despite the early results, the screening program is satisfactory for a program being implemented.

The participation rate of women in our screening program was $33.1 \%$. This value is below rates suggested internationally; these rates range from around $70 \%$ to $80 \%$ coverage in the first round [7-9] as reported in Valencia, where the turnout was $72.98 \%$ [10], to $75 \%$ in the United Kingdom [11]. However, if confronted with other traces that are also in the initial phase, our rate is closer to the $48 \%$ in France [11], $54 \%$ in Australia, and $11.5 \%$ to $54.7 \%$ in different parts of Canada [7]. Another important factor is the incipient experience with breast cancer prevention programs in developing countries like Brazil. We can speculate that if we had the same experience of developed countries, the comparisons would be more relevant.

Most women participants were aged 40 to 49 years (48.2\%), followed by women aged 50 to 59 years (34.4\%) and women aged 60 to 69 years (17.4\%). This is due to the fact that $45.2 \%$ of the eligible population consisted of young women, followed by women aged 50 to 59 years (31.9\%) and, finally, women aged 60 to 69 
years $(22.8 \%)$. The criterion for women selection was the same used by Seguret et al. [11], the American College of Radiology, the American Cancer Society [12], and a major European study [5]. No statistical significance for mammogram examinations in women aged 40 to 49 years nao foi observado quando comparada a faixa etaria de 50 a 59 anos, portanto neste estudo se comprova a validade de realizar o exame de Mamografia em mulheres de 40 a 49 anos.

We found a great demand for the MU for the examinations, which corroborates the premise that use of the $\mathrm{MU}$ is an important tool and facilitated access to mammography [15]. Falta o inicio da frase besides being an important strategy for overcoming obstacles to examinations faced by women in remote areas with poor resources and/or those having difficulty accessing the public health system [15]. A national survey of mammography facilities in North America revealed that $2.4 \%$ of mammograms in the country were performed in an $\mathrm{MU}$, but the number of units assessed was low [16]. On the other hand, England has the prominent presence of more than 90 units, with an annual national coverage of $75 \%[17]$

Generally, the MU has been used in developed countries to allow population subgroups that have some limitations to participating in screening tests for breast cancer to catch up. These subgroups are generally composed of women of low income, low educational profile, and older age who live in rural areas, among other limitations [18-21]. This approach has been also used to aid in organized screening; use of the MU to visit the small counties provides easy access to the mammogram examination and avoids or reduces the transit of the women to generally distant 
cities with appropriated resources for breast examination [22]. Our findings considerably support this assumption, because 10,521 (58.6\%) women sought mammograms in the MU compared with 7443 (41.4\%) women who had the examination in the FU.

Radiologists in the MU conducted 10,521 mammograms, with a daily average of 26.3 examinations and an occupancy rate of $65.7 \%$. The average daily number of examinations was similar to the 31 examinations performed daily in a study of MUs in the United States with an occupancy rate of $15.0 \%$. The same study showed that only $15 \%$ of MUs have an occupancy rate comparable to ours [23]. In regard to the FU, we obtained an occupancy rate of $15.5 \%$, with an average of 18.6 examinations per day. It is clear that the units were not fully occupied to their operational capacity, revealing that it is urgent to implement more intensely a comprehensive interaction between the population and the public health administration in order to appropriately explore the potential of mammography to reduce breast cancer.

The invitation for women to participate in the screening program was carried out by various sources, such as the media, press and radio, letters, lectures, etc. Some of these methods have been already used in developed countries, such as the use of an invitation letter, which offers a participation rate of $85 \%$ [24]. Other programs have combined this strategy with media campaigns, achieving success rates greater than $70 \%$ [10]. The best strategy to promote participation in our study was that related to the primary health services care in public ambulatories and individual reference, especially through the community health agent. This strategy 
was followed by physician influence, which was responsible for persuading $27.0 \%$ of women to have a mammogram. This finding is very important, because a significant percentage of women received the information demanded by their doctors.

The recall rate of screening was $9.4 \%$, which is compatible with internationally recommended values, which are around 10\%[11]. The Breast Cancer Surveillance Consortium, U.S. National Cancer Institute (BCSC); national breast cancer early detection program, U.S. Centers for Disease Control and Prevention (NBCCEDP); and UK National Health Service Breast Screening Program (NHSBSP) had respective rates of recall of $13.1 \%, 11.2 \%$, and $7.4 \%$ for the first round of screening [25].

The positive predictive value for biopsy was low (16\%) compared to the indication of BI-RADS ranging from 25 to $40 \%$; this indicates that many patients referred for biopsy had no lesions, suggesting a conservative approach of the radiologists. Despite the fact that they have well-proven experience in performing diagnostic mammography, apparently they do not feel comfortable dispensing of patients with minimal mammographic alterations.

The program diagnosed 76 cases of breast cancer in the units. The overall detection rate was 4.23 cases per 1,000 examinations. In a large series, the detection rate reported ranged from 8.6 to 10.1 cases per 1,000 examinations [25].

One of the great virtues of screening is to find cases of early stage cancer. We observed that the frequencies of detecting tumors in the early stages of development (EC 0 and EC I) were $48.6 \%$ and $39.1 \%$ in the fixed and mobile units, respectively, representing an overall average of $43.4 \%$ of tumors in these stages. 
The Cancer Hospital of Barretos had a detection rate of $14.5 \%$ for cases of EC 0 and ECI prior to the screening program implementation.

In patients with more advanced breast cancer, we also observed significant improvements. Tumors in advanced stages (CS III and CS IV) accounted for $17 \%$ of cancers detected in the $\mathrm{MU}$ and $14.3 \%$ of cancers detected in the FU, with an overall average detection rate of $15.8 \%$. These values are significantly below the $40.5 \%$ detection rate recorded in the files of Barretos Hospital before the screening program $(p \varangle 0.001)$.

Our findings confirm, in part, the importance of implementing an organized screening program to prevent breast cancer. Among other evidence, the proportion of invasive cases with negative lymph nodes reached a modest $57.4 \%$. The frequency of stage EC 0 and ECI tumors was $43.4 \%$, while the ideal should be $50 \%$. Additionally, it is recommended that $20-25 \%$ of axillary lymph nodes should be positive; however, our results exceeded $42 \%$, which demonstrates the aggressiveness of our cases at diagnosis.

Although we have obtained accurate data and successfully implemented a system of early detection for breast cancer, the major methodological limitation of this study is due to the fact that we failed to separate symptomatic from asymptomatic women. This initiative was carried out based on the philosophy of the PIO XII institution, where the Department of Prevention is included. This initiative was also carried out due to the high demand for mammography in the region, which is confirmed by the high number of referrals from doctors for the 
early detection system. Consequently, the overall deficit is huge because of the lack of a health policy priority for mammographic screening.

However, when we analyzed the breast cancer-positive cases in symptomatic patients, a significant number of these women had undergone early staging. This is important but beyond the main subject of the work. An early detection program as described here was actually deployed, and it is in operational function. Thus far the results are acceptable, with a great potential for progressive improvement. 


\section{REFERENCES}

1. Jemal A, Siegel R, Ward E, Hao Y, Xu J, Thun MJ. Cancer Statistics, 2009. CA A Cancer Journal for Clinicians 2009; 59: 225-249.

2. Parkin DM, Fernandez LM. Use of statistics to assess the global burden of breast cancer. The Breast Journal 2006; 12 Suppl 1: S7080.

3. Parkin DM, Bray F, Ferlay J, Piasini P. Global cancer statistics, 2002. CA A Cancer Journal for Clinicians 2005; 55(2): 74-108.

4. Tabar L, Vitak B, Chen HH, Duffy SW, Yen MF, Chiang CF, et al. The Swedish Two-County Trial twenty years later. Updated mortality results and new insights from long-term follow-up. Radiologic Clinics of North America 2000; 38(4): 625-651.

5. Duffy SW, Tabar L, Chen HH, Holmqvist M, Yen MF, Abdsalah S, et al. The impact of organized mammography service screening on breast carcinoma mortality in seven Swedish counties. Cancer 2002; 95(3): 458-469.

6. Nystrom L, Rutqvist LE, Wall S, Lindgren A, Lindqvist M, Ryden S, et al. Breast cancer screening with mammography: overview of Swedish randomised trials. Lancet 1993; 341(8851): 973-978.

7. Canada H. Quality determinants of organized breast cancer screening programs in Canada. Minister of Public Works and Government Services Canada: Ottawa (ON), 2003. 
8. European guidelines for quality assurance in breast cancer screening and diagnosis. 4th ed. Vol. 1. 2006, Luxembourg: European Communities. 416.

9. American College of Radiology (ACR). ACR BI-RADS Mammography, in ACR breast imaging reporting and data system, breast imaging atlas. 2003, American College of Radiology: Reston, VA.

10. Vizcaino I, Salas D, Vilar JS, Ruiz-Perales F, Herranz C, Ibanez J. Breast cancer screening: first round in the population-based program in Valencia, Spain. Collaborative Group of Readers of the Breast Cancer Screening Program of the Valencia Community. Radiology 1998. 206(1): 253-260.

11. Seguret F, Daures JP, Guizard AV, Mathieu-Daude H, Bonifacj JC, Cherifcheik J, et al. Herault breast screening programme: results after 30 months of a mobile French schedule. European Journal of Cancer Prevention 1995; 4(4): 299-305.

12. Smith RA, Saslow D, Sawyer KA, Burke W, Constanza, ME, Evans WP $3^{\text {rd }}$, et al. American Cancer Society guidelines for breast cancer screening: update 2003. CA A Cancer Journal for Clinicians 2003; 53(3): 141-169.

13. Shapiro S, Coleman EA, Broeders $M$, Codd $M$, de Koning $H$, Fracheboud $\mathrm{J}$, et al. Breast cancer screening programmes in 22 countries: current policies, administration and guidelines. International Breast Cancer Screening Network (IBSN) and the European Network 
of Pilot Projects for Breast Cancer Screening. International Journal of Epidemiology 1998; 27(5): 735-742.

14. Bjurstam N, Bjorneld L, Duffy SW, Smith TC, Cahlin E, Erikson O, et al. The Gothenburg Breast Cancer Screening Trial: preliminary results on breast cancer mortality for women aged 39-49. Journal of the National Cancer Institute. Monographs 1997; (22): 53-55.

15. Dershaw DD, Liberman L, Lippin BS. Mobile mammographic screening of self-referred women: results of 22,540 screenings. Radiology 1992; 184(2): 415-419.

16. Brown ML, Fintor L. U.S. screening mammography services with mobile units: results from the National Survey of Mammography Facilities. Radiology 1995; 195(2): 529-532.

17. Sasieni P. Evaluation of the UK breast screening programmes. Annals of Oncology 2003; 14(8): 1206-1208.

18. Frelix GD, Rosenblatt R, Solomon M, Vikram B. Breast cancer screening in underserved women in the Bronx. Journal of the National Medical Association 1999; 91(4): 195-200.

19. Alexy BB, Elnitsky C. Rural mobile health unit: outcomes. Public Health Nursing 1998; 15(1): 3-11.

20. Levin JR, Hirsch SH, Bastani R, Ganz PA, Lovett ML, Reuben DB. Acceptability of mobile mammography among community-dwelling older women. Journal of the American Geriatrics Society 1997; 45(11): 1365-1370. 
21. Reding DJ, Lappe KA, Krueger M, Kolehouse BL, Steneil D, Leer RA. Cancer screening and prevention in rural Wisconsin: the Greater Marshfield Experience. Wisconsin Medical Journal 1997; 96(8): 32-37.

22. Njor SH, Olsen AH, Bellstrom T, Dyreborg U, Bak M, Axelsson C. Mammography screening in the county of Fyn. November 1993December 1999. APMIS. Supplementum 2003; (110): 1-33.

23. DeBruhl ND, Bassett LW, Jessop NW, Mason AM. Mobile mammography: results of a national survey. Radiology 1996; 201(2): 433-437.

24. Ascunce N, del Moral A, Murillo A, Alfaro C, Apesteguia L, Ros J, et al. Early detection programme for breast cancer in Navarra, Spain. European Journal of Cancer Prevention 1994; 3 Suppl 1: 41-48.

25. Smith-Bindman R, Chu PW, Miglioretti DL, Sickles EA, Blanks R, Ballard-Barbash R, et al. Comparison of screening mammography in the United States and the United kingdom. JAMA: the Journal of the American Medical Association 2003; 290(16): 2129-2137.

26. Humphrey LL, Helfand M, Chan BK, Woolf SH. Breast cancer screening: a summary of the evidence for the U.S. Preventive Services Task Force. Annals of Internal Medicine 2002; 3;137(5 Part 1): $347-360$. 
Table 1. Histological types of breast cancer detected with the screening program

\begin{tabular}{llll}
\hline Histology & \multicolumn{2}{l}{ Site of examination $\mathrm{n}(\%)$} & Total \\
\cline { 2 - 3 } & FU & MU & n (\%) \\
\hline Ductal in situ & $5(6.6)$ & $3(3.9)$ & $8(10.5)$ \\
Invasive ductal & $22(29.0)$ & $33(43.4)$ & $55(72.4)$ \\
Invasive lobular & $8(10.5)$ & $5(6.6)$ & $13(17.1)$ \\
\hline
\end{tabular}

$\mathrm{FU}=$ Fixed unit; $\mathrm{MU}=$ Mobile unit 